Review

\title{
Recent advances of novel thermal combined hot air drying of agricultural crops
}

\author{
Daniel I. Onwude a, b, ${ }^{*}$, Norhashila Hashim ${ }^{\text {a, }{ }^{* *} \text {, Guangnan Chen }}{ }^{c}$ \\ a Department of Biological \& Agricultural Engineering, Faculty of Engineering, Universiti Putra Malaysia, 43400, UPM, Serdang, Selangor, Malaysia \\ ${ }^{\mathrm{b}}$ Department of Agricultural \& Food Engineering, Faculty of Engineering, University of Uyo, 52021, Uyo, Nigeria

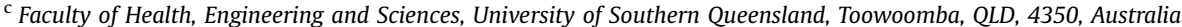

\section{A R T I C L E I N F O}

\section{Article history:}

Received 3 August 2016

Received in revised form

18 September 2016

Accepted 25 September 2016

Available online 30 September 2016

\section{Keywords:}

Novel thermal technology

Hybrid technology

Hurdle technology

Hot air assisted drying

Advanced drying techniques

Drying kinetics

\begin{abstract}
A B S T R A C T
Background: Developing an efficient drying system with combined novel thermal and conventional hotair drying of agricultural crops has become potentially a viable substitute for conventional drying techniques. Due to the synergistic effect, the total energy and time required can be drastically reduced, and the final quality of agricultural crops preserved. The growing interest and research in recent years have already shown that novel thermal with hot-air drying technology can adequately be used in the drying of agricultural crops.

Scope and approach: This review attempts to give a summary of recent advances in the research and applications of novel thermal combined hot-air drying technology for agricultural crops, with particular emphasis on the combination mode, process conditions, process-quality interaction, drying kinetics, energy demand and drying efficiency.

Key findings and conclusions: The combination of novel thermal with hot-air drying provides distinctive opportunities in the development of advanced agricultural crop drying technologies. The most significant advantages of using the above method were the reduction in the drying time and energy consumption as well as, an increase in the drying rate and overall efficiency. More so, the application of infrared and hotair drying on agricultural crops is advantageous in obtaining dried products of better quality. In conclusion, the findings suggest that these technologies have great potentials. Therefore, more studies, especially in their industrial and commercial application are indispensable.
\end{abstract}

๑) 2016 Elsevier Ltd. All rights reserved.

\section{Introduction}

Drying is one of the foremost and often used preservation method. It consists of the removal of moisture from a product, resulting from the simultaneous heat and mass transfer process due to the application of heat (Akpinar, 2006; Hashim, Onwude, \& Rahaman, 2014). It is widely applied in reducing the moisture content (MC) of vegetables, fruits, herbs, grains, spices, oil seeds, wood and other agricultural products with high MC (Chen, 2015). Evidence suggest that drying is also the most important unit operation for most industrial processes (Onwude, Hashim, Janius,

\footnotetext{
* Corresponding author. Department of Biological \& Agricultural Engineering, Faculty of Engineering, Universiti Putra Malaysia, 43400, UPM, Serdang, Selangor, Malaysia.

** Corresponding author.

E-mail addresses: onwudedaniel@uniuyo.edu.ng (D.I. Onwude), norhashila@ upm.edu.my (N. Hashim).
}

Nawi, \& Abdan, 2016a). Chua, Mujumdar, Chou, Hawlader, and Ho (2000) stated that drying is the most energy demanding operation in all industries with about $10-25 \%$ of the total energy. Similarly, Klemes, Smith, and Kim (2008) reported the total energy demand of food industries in most developing countries to be $10-15 \%$. Recently, there is an increasing effort to reduce the postharvest losses of agricultural products, around $30-40 \%$ of total production in developing countries (Karim \& Hawlader, 2005). The drying process may affect (partially or totally) products quality regarding sensory, nutritional and functional attributes. For successful drying operation, the choice of adequate technique, particularly for high-value foods including fruits and vegetables becomes requisite.

There are many conventional drying methods used in postharvest technology including solar drying (El-sebaii \& Shalaby, 2012), osmotic dehydration (Luchese, Gurak, \& Marczak, 2015), vacuum drying (Nadi, Rahimi, Younsi, Tavakoli, \& Hamidi-Esfahani, 2012), hot-air drying (Onwude et al., 2016a, 2016b), fluidized bed 
drying (Sagar \& Suresh Kumar, 2010), and freeze drying (Ciurzyńska \& Lenart, 2011). However, most of these drying techniques involve longer drying time and high amount of energy, resulting in poor quality of the dried products (Moses, Norton, Alagusundaram, \& Tiwari, 2014). Although, Liu, Aziz, Kansha, Bhattacharya, and Tsutsumi (2014, 2012), and Aziz et al. (2011) suggested a new way of improving the existing conventional drying processes based on self-heat recuperation technology, however this method, which focuses mainly on increasing the energy efficiency of the drying processes is complex, and expensive to adapt. Thus, its limitation. In most recent studies, there have been significant developments in using novel techniques, like microwave, radio frequency, infrared, pulse electric field, ultraviolet, ultrasound, ohmic, supercritical, and heat pump heating, in the drying of agricultural crops in terms of pre-treatment, techniques and equipment design that will increase process efficiency and enhance the quality of the final dried products.

Some of these advanced technologies can be applied as pretreatment or in combination with conventional techniques, for decreasing the initial moisture content or changing the tissue structure of crops in a manner that shortens the drying time (Fernandes, Linhares, \& Rodrigues, 2008). In addition, most of these novel techniques produce more superior products, with great reduction in MC, drying time and energy consumption as compared to the conventional drying techniques (Moses et al., 2014).

Novel thermal technologies have recently gained increased interest for preservation processes in food and agro-allied industries (Cullen, Tiwari, \& Valdramidis, 2012; Rastogi, 2012a; Vicente \& Castro, 2007; Witrowa-Rajchert, Wiktor, Sledz, \& Nowacka, 2014). Novel heating technologies, which include microwave (MW), inductive heating $(\mathrm{IH})$, and radio frequency (RF), have been developed to partially or completely replace the conventional method of heating that mainly depends on the convective, conductive and radiative mode of heat transfer (Kaur \& Singh, 2016). These technologies are volumetric forms of heating with a common feature of generating heat directly inside the product (Cullen et al., 2012; Rastogi, 2012a). This has direct implications on energetic, exergetic, and heating efficiency. In addition, infrared (IR) radiation has recently been used to thermally process agricultural crops (Riadh, Ahmad, Marhaban,\& Soh, 2015). Collectively, all these are referred to as novel thermal technologies, where the change in product temperature is the dominant processing factor.

The use of novel thermal techniques to develop drying system have gained considerable attention in recent years (Raghavan et al., 2005; Rawson et al., 2011). Several researchers have shown the integration or combination of these technologies with conventional drying methods. Andrés, Fito, Heredia, and Rosa (2007) studied the combined microwave drying (MWD) and hot-air drying (HAD) of mango products; Mujumdar and Law (2010) reviewed on combined MWD and vacuum drying (VD) of agricultural crops; Soysal, Arslan and Keskin (2009) and Soysal, Ayhan, Eştürk and Arikan (2009) investigated the intermittent MWD and HAD of red pepper and oregano. Using combined novel thermal and conventional hot-air drying technologies, also known as hybrid or hurdle technologies (Cullen et al., 2012), involve successive, intermittent or simultaneous applications of various individual heating techniques with different heat and mass transfer modes. Combined novel thermal and conventional drying techniques are advantageous, particularly because, many individual heating techniques, alone cannot adequately ensure the quality, safety, and stability of dried products. In fact, in some circumstances, the combined drying techniques would allow a milder application of a single heating method with a stronger application of the other heating technique, with consequent improvement in quality of the final products, reduction in energy demand and total drying time, and increase in process efficiency (Andrés et al., 2007; Chong, Figiel, Law, \& Wojdyło, 2013; Moses et al., 2014; Yuan, Tan, Xu, Yuan, \& Dong, 2015).

Overall, although there are significant amounts of scientific literature regarding novel thermal heating, hot-air drying, and hotair assisted drying; it is noticed that most of these studies are carried out in isolation with no assessment on the combination of novel thermal and hot-air drying of agricultural crops, with particular emphasis on the combination modes, drying conditions, application, process-quality interaction, drying kinetics, and energy demand. In view of this, the aim of this review is to highlight some of the latest practical advancements in combined novel thermal and hot-air drying of agricultural crops.

\section{The need for novel drying methods}

Hot-air drying (HAD), in particular, is a widely used method for preserving agricultural crops, especially during industrial food production. This method involves the exposure of a product to a continuous air flow to remove moisture. The theory behind this process is a complex problem because of the different mechanism of heat, mass, water and energy transport process. HAD is naturally harmless and non-toxic, provides a more uniform, hygienic, and rapid dried product that can have extended life of at least a year, however, the quality of hot-air dried products are often drastically reduced (Arslan \& Ozcan, 2011; Zhang et al., 2015; Łechtańska, Szadzińska, \& Kowalski, 2015). In addition, this technique leads to high energy demand and prolonged drying time which could cause severe shrinkage, reduced bulk density, and rehydration capacity, especially at high temperatures (Zhang, Tang, Mujumdar, \& Wang, 2006). Consequently, the combination of novel thermal techniques with HAD method (also referred to as novel thermal assisted drying) has recently gained a significant amount of interest with several publications and reports on theories, design, applications, products and drying kinetics. For example, Praveen Kumar, Hebbar, and Ramesh (2006) studied on the infrared combined HAD of onion slices under different processing conditions. Shi et al. (2008) tested the quality of finished product dried using catalytic infrared (CIR) dryer. The results of their study showed that application of IR resulted in a reduced drying time when compared to results of HAD

Furthermore, the use of combined IR radiation and hot-air (HA) heating is reported to improve heating efficiency when compared to radiation or HA heating alone, because of its synergistic effect (Hebbar, Vishwanathan, \& Ramesh, 2004). Datta and Ni (2002) studied the combined IR, microwave (MW) and HAD of food. Afzal, Abe, and Hikida (1999) demonstrated the effect of combined far-infrared (FIR) and HAD on the energy demand of barley and found a $245 \%$ reduction in the total energy required when compared to results of $\mathrm{HAD}$ at $70{ }^{\circ} \mathrm{C}$.

Most agricultural crops, especially fruits and vegetables, contains high MC, thus can be effectively dried using the combination of two or more different drying methods, because it provides the synergistic effect (Fig. 1.), reduces total energy requirement, and

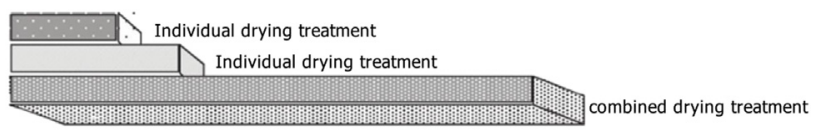

Synergistic effect

Preservation effect \& Efficiency

Fig. 1. Synergistic effect obtained by combining different drying technologies (Adapted from: Raso \& Barbosa-Cánovas, 2003). 
reduces drying time while retaining most quality parameters. Considering the increased complexities and different agricultural crops, there is no single general drying method suitable for every product. However, there have been significant advances in recent years, regarding approaches that can increase the efficiency of conventional drying methods which show commercial promise. For instance, during IR combined HAD or MW combined HAD, the rapid heating of agricultural products by either IR or MW radiation increases the rate of water transport to the surface, while the water vapour formed at the surface is swiftly removed by convective airflow.

\section{Novel thermal and hot-air combined drying}

The drying of agricultural crops is often controlled by mechanisms such as diffusion, and capillary action that occurs in the porous region (Erbay \& Icier, 2010; Onwude et al., 2016a). This can lead to time constraints due to a high amount of internal resistance in the product. This resistance can be surmounted with the help of other heating technologies. A recent study by Moses et al. (2014) showed that combined novel thermal technology and HAD resulted in significant drying time reduction, increased drying efficiency, and superior product quality as compared to HAD alone. The examples covered below include MW combined HAD drying and IR combined HAD, which are well documented in the literature for the past 9 years. Recent advances in combined RF and HAD were also covered.

\subsection{Microwave and hot-air combined drying}

Electromagnetic (EM) radiation comprises alternating electric and magnetic waves. The EM waves cover a wide range of spectrum from low-frequency telecommunication, microwaves (MWs), IR, to gamma radiation. MWs lies in the EM spectrum with frequency from $300 \mathrm{MHz}$ to $300 \mathrm{GHz}$ (Wray \& Ramaswamy, 2015). These travelling waves proliferate with a distinct measure of time between the highest points during oscillation, ranging from 331028 to 3310211s (Venkatesh \& Raghavan, 2004). Coincidentally, several molecular transitions including molecular dissociation, various reactions in water and essentially, dipole and ionic relaxation in water occur within this range. According to Miura, Yagihara, and Mashimo (2003), the dipole and ionic relaxation of water can change from a small-amplitude, high-frequency process around $100 \mathrm{MHz}$ for bound water to $18 \mathrm{GHz}$ for pure water. Consequently, the dielectric property is often studied when dealing with the heating effects of MWs.

Moreover, when MW strikes a product, some energy is transmitted, parts of which are reflected while others are absorbed by the product. The absorbed energy is dissipated as heat, which is also because of the interaction of the electric field and bounded charged particle within the product. In perspective, the electric field component caused the movement of bounded charged particle within the product until equilibrium is attained between opposing and electric forces. Consequently, a dipolar polarization is formed within the product. The process of molecular movement within the product is particularly fast due to the high electrical field frequency. For example, there is 2.45 billion cycles/second change of polarity at MW frequency of $2450 \mathrm{MHz}$. Subsequently, an intense heat is created which can grow rapidly as much, as $10^{\circ} \mathrm{C}$ per second (Rastogi, 2012a, 2012b). This heating process results in volumetric heating of the product (Miura et al., 2003).

The MW drying (MWD) mechanism involves exposing the product to MW radiation, which penetrates directly into the product, causing a rapid internal volumetric heating, as a result of the friction generated by rotating dipoles and ion movement in the drying product (Miura et al., 2003; Sadeghi, Mirzabeigi Kesbi, \& Mireei, 2013). In addition, during this process, mass transfer occurs due to the generation of vapour within the product, forcing the water vapour to the surface of the product hence, moisture can easily be removed. During combined MW and HAD (Fig. 2.), the established moisture at the surface of the product due to MW heat can then be removed rapidly from the surface to the atmosphere by convective air flow with minimal energy requirement. (Amiri Chayjan, Kaveh, \& Khayati, 2014; Kaur \& Singh, 2014; Sadeghi et al., 2013; Soysal, Arslan et al., 2009; Soysal, Ayhan et al. 2009; Zhang et al., 2006). This method increases drying rate, efficiency and considerably reduces drying time. Mostly, the ability of MW radiation to penetrate the product during combined MW and HAD can lead to controlled, and precise heating, which improves the drying rate, drying time and quality of agricultural crops.

Zhang et al. (2006) described 3 different MW and HAD combination sequence: (1) They identified the application of the MW energy at the start of the drying process, whereby the product's interior is heated rapidly, causing moisture movement towards the surface of the product and thereby enabling the easy removal of the water vapour at the surface to the atmosphere, by the application of hot air (MW + HAD). Amiri Chayjan et al. (2014) used this combination mode (MW + HAD) for the drying of hawthorn. Similarly, in recent times, several researchers have applied this combination mode in the drying of other agricultural crops such as papaya (Yousefi, Niakousari, \& Moradi, 2013), Thompson seedless grape (Kassem, Shokr, El-Mahdy, Aboukarima, \& Hamed, 2011) and canola seeds (Hemis, Choudhary, Gariepy, \& Raghavan, 2015); (2) by applying MW energy after the initial period of constant rate. Here, the surface of the product is dry and large portion of the moisture is situated at the center of the product. At this stage, the heat is generated internally, and the vapour pressures thereof causes the movement of moisture to the surface of the product. The hot air is later introduced after the first falling rate period (IMW + HAD). During the intermittent change of the air flux, the product experiences relaxation due to the constant change in the flow of air, and the concentration gradients of water will decrease. Water diffuses to the surface and is readily dispersed to the atmosphere on introducing airflow. Soysal, Arslan et al. (2009); Soysal, Ayhan et al.(2009) investigated the drying of Oregon and Red pepper using similar approach to IMW + HAD. A similar report on apple fruit has also been investigated (Aghilinategh et al., 2015); (3) by applying MW during the second falling rate period or the end of the entire drying process, when MC is low (HAD + MW). During this process, agricultural crops experiences structural shrinkage which in turn controls the rate of water movement (diffusion), thus resulting in drying rates reduction. Conversely, during MW + HAD, shrinkage of tissue structure can be prevented. Sometimes, applying HAD + MWD may enhance the removal of bound water from the product (Fang et al., 2011; Kaur \& Singh, 2014). This combination mode has been used in the drying of Thompson seedless grape (Kassem et al., 2011) and beetroot (Kaur \& Singh, 2014).

In addition to the above combination modes, simultaneous MW and hot-air drying process (MW-HAD) has also been studied extensively, with different temperature, power air velocity, and modelling combinations. During simultaneous MW and hot-air drying (MW-HAD), heating is immediate and fast as a result of sufficient energy transfer within the product, and convective heat and mass transfer; hence the constant rate period and surfacecenter conduction stage are largely eliminated. As drying continues, additional energy input causes internal generation of heat, increasing internal temperature, mass transfer and vapour pressure, which results in moisture transfer to the surface, thus increasing the drying rate (Seremet (Ceclu), Botez, Nistor, 


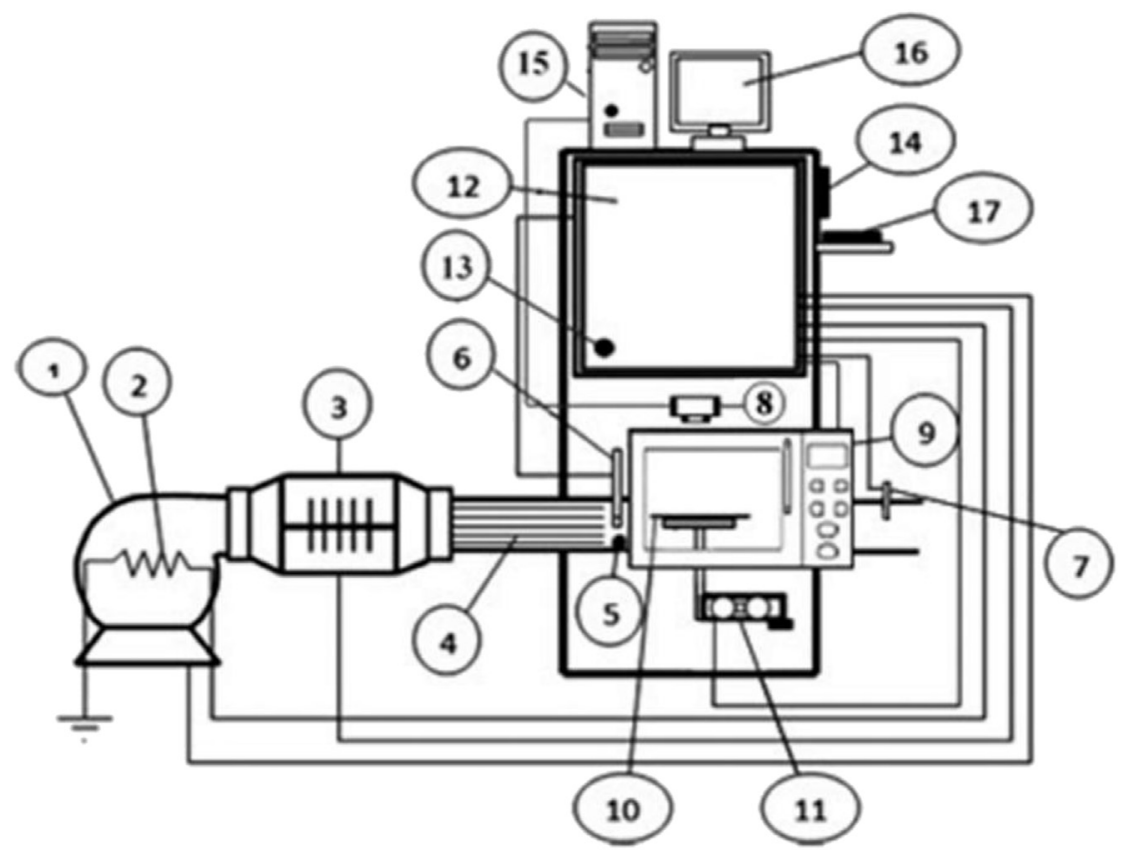

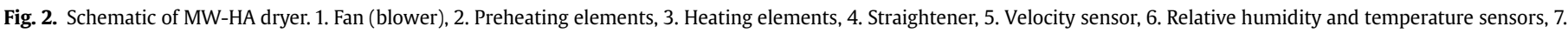

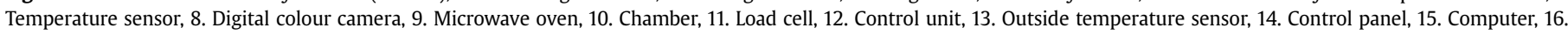
Monitor, 17. Keyboard (Adapted from: Aghilinategh et al., 2015).

\section{Andronoiu \& Mocanu, 2015).}

Several studies have shown the benefits of using combined MW and HAD of agricultural crops; a list of recent publications in the past 9 years is presented in Table 1. From the table, MW and HA combined drying modes are presented in terms of simultaneous (MW-HAD), which was the most used combination mode, sequential (MW + HAD; HAD + MW) and intermittently (IMW + HAD).

\subsubsection{Application to agricultural crops}

3.1.1.1. Cereals. Table 1 summarizes some of the findings on MW and HA combined drying of cereals in the past 9 years. Jiao, $\mathrm{Xu}$, and Jin (2014) studied on the drying and rehydration of hybrid indica rice in a combined MW-HAD. They mentioned that there was no constant rate period during the drying process. As expected, the drying rate of MW - HAD was higher than that for MW alone, and much greater than HAD. Moreover, the combined drying process required only $25 \%$ of the total drying time of HAD and MW alone (3fold faster than HAD). The optimum drying conditions were identified to be MW power of $300 \mathrm{~W}$, and $80^{\circ} \mathrm{C}$.

Similarly, Gowen, Abu-Ghannam, Frias, and Oliveira (2008) applied MW-HAD in the drying of soybeans. They observed an increased drying rate at the beginning of the entire drying process and a subsequent decrease in the drying rate. The increase in the drying rate at the initial stage is due to "warming-up" of the sample, when mass losses are small, and due to the short drying duration, whereas the subsequent reduction in drying rate is due to the drying out of the sample surface following water evaporation from the surface of the sample. The best result based on colour, MC, RC and drying time was recorded at $210 \mathrm{~W}-160{ }^{\circ} \mathrm{C}$ and the rehydration time was $50-60 \%$ less than HAD or MW alone, respectively.

3.1.1.2. Fruits and vegetables. Varith, Dijkanarukkul, Achariyaviriya, and Achariyaviriya (2007) applied combined MW-HAD on peeled longan. The results of their study showed a unique convexedshaped drying rate before falling rate period. This phenomenon is due to the practical increase in moisture concentration due to MW power. In addition, this shows a small shrinkage occurrence and high porosity during the drying process. The drying time during MW-HAD was reduced by $64.3 \%$ and energy consumption by $48.2 \%$ as compared to results of HAD alone. The best drying efficiencies were $40{ }^{\circ} \mathrm{C}$ and $450 \mathrm{~W}$-MW for $1.7 \mathrm{~h}$ followed by $60^{\circ} \mathrm{C}$ with $300 \mathrm{~W}$ MW for $3.3 \mathrm{~h}$.

Sadeghi et al. (2013) reported the findings on combined MWHAD drying of Lemon. In the initial drying stage under this combination mode, a short initial drying rate and only 1 falling rate period were observed (Fig. 3c.), as compared to those of HAD alone (Fig. 3a) and MWD alone (Fig. 3b). This delay is the warming-up period, which occurs because of the short drying time experienced during this period for combined MW-HAD. In perspective, lemon fruit contains high moisture content which enables higher absorption of MW at the initial drying stage, and therefore, higher drying rates. In addition, during this combined MW-HAD drying process, the drying time reduced $17-31$ times as compared to HAD. Similarly, Mirzabeigi Kesbi et al. (2016) demonstrated that the drying duration of lemon slices was considerably reduced about 20-30 times when using combined MW-HAD as compared to HAD, due to the volumetric heating caused by MW energy, consequently producing an outward flux of rapidly escaping vapour, hence an increase in drying rate. Drying rate curve indicated only a falling rate period with a short warming-up period at the beginning of the process. Improvement in quality parameters of lemon, when compared with drying using HAD alone, was also observed.

Furthermore, in the drying of oranges, Talens, Castro-giraldez, and Fito (2016) reported the dominant drying mechanism of surface and evaporation, and MW dissipation with penetration are coupled (HAD shrinkage and MW swelling). In the same vein, Alibas (2007) also studied the combined MW-HAD drying of pumpkin. It was observed that drying time of MW-HAD at $160 \mathrm{~W}-$ $50{ }^{\circ} \mathrm{C}$ was 1.48 times shorter than MWD and, 3.2 times shorter than HAD $\left(50^{\circ} \mathrm{C}\right)$. Accordingly, the optimum MW-HAD combination is $350 \mathrm{~W}-75^{\circ} \mathrm{C}$ with energy consumption of $0.29 \mathrm{kWh}$ and drying 
Table 1

Published data on combined microwave and hot-air drying of agricultural crops in the past 9 years.

\begin{tabular}{|c|c|c|c|c|c|}
\hline Agricultural crops & $\begin{array}{l}\text { Combination } \\
\text { mode }\end{array}$ & Drying process conditions & Significant findings & Modelling approach & References \\
\hline $\begin{array}{l}\text { Apple (Red } \\
\text { delicious) }\end{array}$ & $\begin{array}{l}\text { IMW+HAD; } \\
\text { MW-HAD }\end{array}$ & $\begin{array}{l}\mathrm{h}=6 \mathrm{~mm} ; \mathrm{v}=0.5-2 \mathrm{~m} / \mathrm{s} \\
\mathrm{P}=200-600 \mathrm{~W} ; \mathrm{PR}=2-6\end{array}$ & $\begin{array}{l}\text { MW-HAD had } 92 \% \text { reduction in the drying } \\
\text { time; } D_{\text {eff }}=1.92 \times 10^{-8}-1.58 \times 10^{-7} ; E_{a}=4.14 \mathrm{~W} / \\
\mathrm{g}\end{array}$ & - & $\begin{array}{l}\text { Aghilinategh } \\
\text { et al. (2015) }\end{array}$ \\
\hline $\begin{array}{l}\text { Apples (Golden } \\
\text { delicious) }\end{array}$ & MW-HAD & $\begin{array}{l}\mathrm{d}=2.2 \mathrm{~mm} ; \mathrm{h}=4 \mathrm{~mm} ; \mathrm{v}=2 \mathrm{~m} / \\
\mathrm{s} ; \mathrm{RH}=35 \% ; \mathrm{T}=50^{\circ} \mathrm{C} ; \\
\mathrm{P}=300 \mathrm{~W}\end{array}$ & $\begin{array}{l}\text { Reduced drying time by } 20 \% \text {; less shrinkage } \\
\text { effect }\end{array}$ & - & $\begin{array}{l}\text { Askari et al. } \\
(2009)\end{array}$ \\
\hline $\begin{array}{l}\text { Banana (Nendran } \\
\text { Spp) }\end{array}$ & MW-HAD & $\begin{array}{l}\mathrm{h}=1-3 \mathrm{~mm} ; \mathrm{v}=1.6 \pm 0.2 \mathrm{~m} / \mathrm{s} \\
\mathrm{T}=40^{\circ} \mathrm{C} ; \mathrm{P}=100 \mathrm{~W}\end{array}$ & Reduced drying time up to $94 \%$. & $\begin{array}{l}\text { Semi theoretical Midilli } \\
\text { et al. model }\end{array}$ & $\begin{array}{l}\text { Ganesapillai } \\
\text { et al. (2011) }\end{array}$ \\
\hline $\begin{array}{l}\text { Beetroots } \\
\quad(\text { B. Vulgaris L.) }\end{array}$ & $\mathrm{HAD}+\mathrm{MW}$ & $\begin{array}{l}\mathrm{h}=3 \mathrm{~mm} ; \mathrm{T}=55-75^{\circ} \mathrm{C} \\
\mathrm{P}=540-1080 \mathrm{~W}\end{array}$ & Drying time saving of up to $44.2 \%$ & $\begin{array}{l}\text { Semi theoretical Two- } \\
\text { terms model; Semi } \\
\text { theoretical Logarithm } \\
\text { model }\end{array}$ & $\begin{array}{l}\text { Kaur and Singh } \\
\text { (2014) }\end{array}$ \\
\hline $\begin{array}{l}\text { Broccoli stalk } \\
\quad \text { (Brassica oleracea } \\
\text { L. Var. Italica) }\end{array}$ & MW-HAD & $\begin{array}{l}\mathrm{h}=6 \mathrm{~mm} ; \mathrm{d}=23 \mathrm{~mm} ; \\
\mathrm{v}=1.4 \mathrm{~m} / \mathrm{s} ; \mathrm{P}=100 \mathrm{~W} ; \mathrm{T}=40 \\
-60{ }^{\circ} \mathrm{C}\end{array}$ & $\begin{array}{l}\text { Drying time of MW-HAD reduced by } 42-55 \% \text {; } \\
D_{\text {eff }}=6.64 \times 10^{-8}-13.31 \times 10^{-8} \mathrm{~m}^{2} / \mathrm{s}\end{array}$ & $\begin{array}{l}\text { Semi theoretical Lewis } \\
\text { model }\end{array}$ & $\begin{array}{l}\text { Horrungsiwat } \\
\text { et al. (2016) }\end{array}$ \\
\hline $\begin{array}{l}\text { Canola Seed } \\
\text { (Brassica napus; } \\
\text { Brassica rapa) }\end{array}$ & MW + HAD & $\mathrm{T}=40-60{ }^{\circ} \mathrm{C} ; \mathrm{P}=0-750 \mathrm{~W}$ & $97 \%$ increase in the drying rate & $\begin{array}{l}\text { Coupled mathematical } \\
\text { model }\end{array}$ & $\begin{array}{l}\text { Hemis et al. } \\
(2015)\end{array}$ \\
\hline $\begin{array}{l}\text { Citrus lemon (Citrus } \\
\quad \text { limon L.) }\end{array}$ & MW - HAD & $\begin{array}{l}\mathrm{h}=5 \pm 1 \mathrm{~mm} ; \mathrm{v}=1 \mathrm{~m} / \mathrm{s} \\
\mathrm{T}=22^{\circ} \mathrm{C} ; \mathrm{P}=180-720 \mathrm{~W}\end{array}$ & - & $\begin{array}{l}\text { Semi theoretical Midilli } \\
\text { et al. model }\end{array}$ & $\begin{array}{l}\text { Darvishi et al. } \\
\text { (2014) }\end{array}$ \\
\hline $\begin{array}{l}\text { Green pepper } \\
\quad \text { (Capsicum } \\
\text { annuum L.) }\end{array}$ & $\begin{array}{l}\text { MW-HAD } \\
\text { (simultaneous } \\
\text { and periodical) }\end{array}$ & $\begin{array}{l}\mathrm{L}=3 \mathrm{~cm} ; \mathrm{W}=2 \mathrm{~cm} \\
\mathrm{v}=1.8 \pm 0.1 \mathrm{~m} / \mathrm{s} ; \mathrm{T}=75^{\circ} \mathrm{C} \\
\text { Effective } \mathrm{P}=62 \mathrm{~W}\end{array}$ & $\begin{array}{l}\text { MW-HAD had the lowest energy consumption } \\
\text { of } 3.3 \pm 0.1 \mathrm{kWh}, 60 \% \text { reduction compared with } \\
\text { HAD; MW-HAD had the highest vitamin C } \\
\text { retention of } 63.9 \% \text { as compared to } 46.54 \% \text { of } \\
\text { HAD }\end{array}$ & - & $\begin{array}{l}\text { Łechtańska } \\
\text { et al. (2015) }\end{array}$ \\
\hline Hawthorn & $\mathrm{MW}+\mathrm{HAD}$ & $\begin{array}{l}\mathrm{P}=270-630 \mathrm{~W} ; \mathrm{V}=0.4 \\
-1.6 \mathrm{~m} / \mathrm{s} ; \mathrm{T}=40-70{ }^{\circ} \mathrm{C}\end{array}$ & $\begin{array}{l}63.39 \% \text { shrinkage at } 70{ }^{\circ} \mathrm{C}, 0.4 \mathrm{~m} / \mathrm{s} \text { and } 630 \mathrm{~W} \text {; } \\
\text { Specific energy consumption ranges from } \\
1343.14 \mathrm{MJ} / \mathrm{K}-148.85 \mathrm{MJ} / \mathrm{K} ; D_{\text {eff }}=8.81 \times 10^{-9} \\
-9.29 \times 10^{-10} \mathrm{~m}^{2} / \mathrm{s} ; E_{a}=27.9-12.25 \mathrm{KJ}\end{array}$ & $\begin{array}{l}\text { Semi-theoretical Midilli } \\
\text { et al. model }\end{array}$ & $\begin{array}{l}\text { Amiri Chayjan } \\
\text { et al. (2014) }\end{array}$ \\
\hline \multirow[t]{2}{*}{ Lemon } & MW-HAD & $\begin{array}{l}\mathrm{h}=5 \mathrm{~mm} ; \mathrm{d}=5 \mathrm{~mm} ; \mathrm{T}=50 \\
-60^{\circ} \mathrm{C} ; \mathrm{P}=185.5 \text { and } 388.5 \mathrm{~W} \\
(\text { specific } \mathrm{P}=0.97 \text { and } \\
\left.2.04 \mathrm{Wg}^{-1}\right)\end{array}$ & $\begin{array}{l}\text { Reduced drying time of about } 20-30 \text { when } \\
\text { compared with using HAD alone }\end{array}$ & $\begin{array}{l}\text { Semi theoretical Midilli } \\
\text { et al. model }\end{array}$ & $\begin{array}{l}\text { Mirzabeigi } \\
\text { Kesbi, Sadeghi, } \\
\text { and Mireei } \\
(2016)\end{array}$ \\
\hline & MW-HAD & $\begin{array}{l}\mathrm{h}=5 \mathrm{~mm} ; \mathrm{d}=50 \pm 3 \mathrm{~mm} \\
\mathrm{~T}=50-60{ }^{\circ} \mathrm{C} ; \mathrm{R} . \mathrm{H}=20-95 \% \\
\mathrm{P}=185.5 \text { and } 388.5 \mathrm{~W} \text { (Specific } \\
\mathrm{P}=0.97 \text { and } 2.04 \mathrm{Wg}-1 \\
\text { respectively) }\end{array}$ & $\begin{array}{l}\text { MW-HAD }=17-31 \text { times reduction in drying } \\
\text { time; } D_{\text {eff }} \text { increased } 21-38 \text { times as compared } \\
\text { to HAD; } D_{\text {eff }}=4.116 \times 10^{-9}-7.618 \times 10^{-10} \mathrm{~m}^{2} / \\
\mathrm{s}\end{array}$ & $\begin{array}{l}\text { Diffusion models } \\
\text { (Dincer and Dost; Crank } \\
\text { models) }\end{array}$ & $\begin{array}{l}\text { Sadeghi et al. } \\
\text { (2013) }\end{array}$ \\
\hline Longan & MW-HAD & $\begin{array}{l}\mathrm{T}=40-60{ }^{\circ} \mathrm{C} ; \mathrm{P}=100-450 \mathrm{~W} \\
\mathrm{v}=0.7 \mathrm{~m} / \mathrm{s}\end{array}$ & Reduced drying time by $64.3 \%$; Energy by $48.2 \%$ & - & $\begin{array}{l}\text { Varith et al. } \\
\text { (2007) }\end{array}$ \\
\hline $\begin{array}{l}\text { Moringa Olifera } \\
\text { (fruits) }\end{array}$ & MW-HAD & $\begin{array}{l}\mathrm{L}=10 \mathrm{~mm} ; \mathrm{d}=10 \mathrm{~mm} ; \mathrm{T}=50 \\
-70{ }^{\circ} \mathrm{C} ; \mathrm{P}=0-750 \mathrm{~W} ; \mathrm{P} \\
\text { density }=1 \mathrm{~W} / \mathrm{g}\end{array}$ & MW-HAD at $60^{\circ} \mathrm{C}$ with $1 \mathrm{~W} / \mathrm{g}$ was the optimum & $\begin{array}{l}\text { Semi theoretical Page } \\
\text { model }\end{array}$ & $\begin{array}{l}\text { Dev et al. } \\
\text { (2011) }\end{array}$ \\
\hline $\begin{array}{l}\text { Mushrooms } \\
\qquad \text { (A. bisporus) }\end{array}$ & MW-HAD & $\begin{array}{l}\mathrm{d}=1-5 \mathrm{~mm} ; \mathrm{v}=2 \mathrm{~m} / \mathrm{s} \\
\mathrm{RH}=35 \% ; \mathrm{T}=50^{\circ} \mathrm{C} ; \mathrm{P}=300 \mathrm{~W}\end{array}$ & Reduced drying time by $34 \%$ & - & $\begin{array}{l}\text { Askari et al. } \\
(2009)\end{array}$ \\
\hline $\begin{array}{l}\text { Mushrooms } \\
\quad \text { (Lentinus edodes) }\end{array}$ & MW-HAD & $\begin{array}{l}\mathrm{T}=60^{\circ} \mathrm{C} ; \mathrm{v}=1 \mathrm{~m} / \mathrm{s} ; \mathrm{P}=600 \mathrm{~W} \\
\text { Power density }=4 \mathrm{~W} / \mathrm{g}\end{array}$ & $\begin{array}{l}\text { Reduced drying time by } 80 \% \text { when compared } \\
\text { with HAD; }\end{array}$ & & $\begin{array}{l}\text { Wang et al. } \\
(2014 a, b)\end{array}$ \\
\hline $\begin{array}{l}\text { Oranges (Citrus } \\
\text { sinensis) }\end{array}$ & MW-HAD & $\begin{array}{l}\mathrm{T}=55^{\circ} \mathrm{C} ; \mathrm{P}=2000 \mathrm{~W} \\
\mathrm{v}=2.5 \mathrm{~m} / \mathrm{s} ; \text { Energy }=0-6 \mathrm{w} / \mathrm{g}\end{array}$ & - & - & $\begin{array}{l}\text { Talens et al. } \\
\text { (2016) }\end{array}$ \\
\hline $\begin{array}{l}\text { Oregano (O. vulgare } \\
\quad \text { L. ssp. hirtum) }\end{array}$ & $\begin{array}{l}\text { IMW+HAD; } \\
\text { MW-HAD }\end{array}$ & $\begin{array}{l}\mathrm{T}=40-50{ }^{\circ} \mathrm{C} ; \mathrm{P}=700 \mathrm{~W} \text {; The } \\
\text { on-off timings used in the } \\
\mathrm{IMW}+\mathrm{HAD}=15 \mathrm{~s} \text { on } 30 \mathrm{~s} \text { off } \\
\left(\mathrm{PR}^{1 / 43.0)}, 15 \mathrm{~s} \text { on } 45 \mathrm{~s} \text { off }\right. \\
\left(\mathrm{PR}^{1 / 44.0)} \text {, and } 15 \mathrm{~s} \text { on } 60 \mathrm{~s} \text { off }\right. \\
\left(\mathrm{PR}^{1} / 45.0\right)\end{array}$ & $\begin{array}{l}\text { ADR }=29.5-59.0 \text { times higher than HAD alone, } \\
\text { 3.5-5.7 times higher than IMW }+ \text { HAD; Specific } \\
\text { energy consumption }(\mathrm{SEC}), \mathrm{MW}-\mathrm{HAD}=12.7 \\
-14.0 \text { times lower than HAD; IMW }+\mathrm{HAD}=4.7 \\
-11.2 \text { times more efficient than HAD alone. }\end{array}$ & - & $\begin{array}{l}\text { Soysal, Arslan, } \\
\text { et al. (2009) }\end{array}$ \\
\hline $\begin{array}{l}\text { Papaya (Carica } \\
\text { papaya } \mathrm{L} .)\end{array}$ & $\mathrm{MW}+\mathrm{HAD}$ & $\begin{array}{l}\mathrm{h}=5 \pm 1 \mathrm{~mm} \\
\mathrm{AH}=0.6 \pm 0.02 \mathrm{~g} / \mathrm{kg} \text { (dry air); } \\
\mathrm{v}=0.9 \pm 0.1 \mathrm{~m} / \mathrm{s} ; \mathrm{T}=40-60{ }^{\circ} \mathrm{C} \\
\mathrm{P}=180-900 \mathrm{~W} ; \text { nominal } \\
\mathrm{P}=540 \mathrm{~W}\end{array}$ & - & $\begin{array}{l}\text { Semi theoretical Page } \\
\text { model, and Two-term } \\
\text { model }\end{array}$ & $\begin{array}{l}\text { Yousefi et al. } \\
\text { (2013) }\end{array}$ \\
\hline Potatoes & MW-HAD & $\begin{array}{l}\mathrm{L}=43 \mathrm{~mm} ; \mathrm{W}=50 \mathrm{~mm} \\
\mathrm{~h}=100 \mathrm{~mm} ; \mathrm{v}=4.5 \mathrm{~m} / \mathrm{s} \\
\mathrm{T}=46.85^{\circ} \mathrm{C} ; \mathrm{P}=300 \mathrm{~W}\end{array}$ & $\begin{array}{l}\text { Effective drying using MW-HAD depends on the } \\
\text { dielectric properties of the material }\end{array}$ & $\begin{array}{l}\text { Multi-physics } \\
\text { modelling; Maxwell } \\
\text { equation }\end{array}$ & $\begin{array}{l}\text { Malafronte } \\
\text { et al. (2012) }\end{array}$ \\
\hline $\begin{array}{l}\text { Pumpkin (Cucurbita } \\
\text { maxima) }\end{array}$ & MW-HAD & $\begin{array}{l}\mathrm{h}=5 \mathrm{~mm} ; \mathrm{L}=40 \mathrm{~mm} \\
\mathrm{~W}=20 \mathrm{~mm} ; \mathrm{v}=1 \mathrm{~m} / \mathrm{s} ; \mathrm{T}=50 \\
-75^{\circ} \mathrm{C} ; \mathrm{P}=160-350 \mathrm{~W}\end{array}$ & $\begin{array}{l}\text { The optimum combination is } 350 \mathrm{~W}-75^{\circ} \mathrm{C} \text { with } \\
\text { energy consumption of } 0.29 \mathrm{kWh} \text { and drying } \\
\text { time of } 31 \mathrm{~min} \text {. }\end{array}$ & $\begin{array}{l}\text { Semi theoretical Page } \\
\text { model }\end{array}$ & Alibas (2007) \\
\hline $\begin{array}{l}\text { Red pepper } \\
\quad \text { (Capsicum } \\
\text { annuum L.) }\end{array}$ & $\begin{array}{l}\text { IMW+HAD; } \\
\text { MW-HAD }\end{array}$ & $\begin{array}{l}\mathrm{L}=2.5 \mathrm{~cm} ; \mathrm{W}=2.0 \mathrm{~cm} ; \mathrm{T}=35 \\
-75^{\circ} \mathrm{C} ; \mathrm{P}=697.9-597.2 \\
\mathrm{PR}=0.0-4.0\end{array}$ & $\begin{array}{l}\text { HAD drying time was } 10.4-19.6 \text { times longer } \\
\text { than MW-HAD and } 2.5-11.8 \text { times longer than } \\
\text { the IMW + HAD }\end{array}$ & - & $\begin{array}{l}\text { Soysal, Ayhan, } \\
\text { et al. (2009) }\end{array}$ \\
\hline Rice (Hybrid Indica) & MW-HAD & $\begin{array}{l}\mathrm{V}=1 \pm 0.05 \mathrm{~m} / \mathrm{s} ; \mathrm{T}=70-90^{\circ} \mathrm{C} \\
\mathrm{P}=210-560 \mathrm{~W}\end{array}$ & Optimum MW-HAD condition $=300$, and $80^{\circ} \mathrm{C}$ & $\begin{array}{l}\text { Semi theoretical Page } \\
\text { model }\end{array}$ & Jiao et al. (2014) \\
\hline Soybeans & MW-HAD & $\begin{array}{l}\mathrm{v}=1 \pm 0.05 \mathrm{~m} / \mathrm{s} ; \mathrm{T}=160{ }^{\circ} \mathrm{C} \\
-200{ }^{\circ} \mathrm{C} ; \mathrm{P}=210-560 \mathrm{~W}\end{array}$ & $\begin{array}{l}\text { The best result in terms of colour, MC, RC and t } \\
\text { was recorded at } 210 \mathrm{~W}-160{ }^{\circ} \mathrm{C} \text {; Rehydration }\end{array}$ & $\begin{array}{l}\text { Semi theoretical Page } \\
\text { model }\end{array}$ & $\begin{array}{l}\text { Gowen et al. } \\
\text { (2008) }\end{array}$ \\
\hline
\end{tabular}


Table 1 (continued)

\begin{tabular}{|c|c|c|c|c|c|}
\hline Agricultural crops & $\begin{array}{l}\text { Combination } \\
\text { mode }\end{array}$ & Drying process conditions & Significant findings & Modelling approach & References \\
\hline & & & $\begin{array}{l}\text { time was } 50 \& 60 \% \text { less than HAD \& MW alone, } \\
\text { respectively. }\end{array}$ & & \\
\hline Strawberries & MW-HAD & $\begin{array}{l}\mathrm{d}=2.2 \mathrm{~mm} ; \mathrm{v}=2 \mathrm{~m} / \mathrm{s} \\
\mathrm{RH}=35 \% ; \mathrm{T}=40^{\circ} \mathrm{C} ; \mathrm{P}=300 \mathrm{~W}\end{array}$ & $\begin{array}{l}\text { Reduced drying time by } 30 \% \text {; less shrinkage } \\
\text { effect }\end{array}$ & - & $\begin{array}{l}\text { Askari et al. } \\
\text { (2009) }\end{array}$ \\
\hline $\begin{array}{l}\text { Thompson seedless } \\
\text { grape }\end{array}$ & $\begin{array}{l}\text { MW + HAD } \\
\mathrm{HAD}+\mathrm{MW}\end{array}$ & $\mathrm{T}=70^{\circ} \mathrm{C} ; \mathrm{P}=75-900 \mathrm{~W}$ & $\begin{array}{l}\text { Optimum selection percentage (SP } \% \text { ): } \\
\text { MW }+\mathrm{HAD}=78, \mathrm{HAD}+\mathrm{MW}=67, \mathrm{HA}=56 \text {; } \\
\text { The best combination was observed to be } \\
\text { MW + HAD }\end{array}$ & $\begin{array}{l}\text { Semi theoretical Page } \\
\text { model }\end{array}$ & $\begin{array}{l}\text { Kassem et al. } \\
\text { (2011) }\end{array}$ \\
\hline $\begin{array}{l}\text { Tomatoes (var. } \\
\text { Marglobe) }\end{array}$ & MW-HAD & $\begin{array}{l}\mathrm{h}=5 \mathrm{~mm} ; \mathrm{v}=1 \mathrm{~m} / \mathrm{s} ; \mathrm{T}=40 \\
-80^{\circ} \mathrm{C} ; \text { Power density }=1.13 \\
-3.11 \mathrm{~W} / \mathrm{g}\end{array}$ & $\begin{array}{l}\text { 84\% reduction in drying time to } 3.3 \mathrm{~h} \text { for a safe } \\
\text { moisture at } 1.13 \mathrm{~W} / \mathrm{g}-50{ }^{\circ} \mathrm{C} \text {; The optimum } \\
\text { drying parameter }=1.13 \mathrm{~W} / \mathrm{g} \text { and } 50^{\circ} \mathrm{C} \text {. }\end{array}$ & $\begin{array}{l}\text { Semi theoretical Page } \\
\text { model }\end{array}$ & $\begin{array}{l}\text { Workneh and } \\
\text { Oke (2013) }\end{array}$ \\
\hline $\begin{array}{l}\text { Tomatoes } \\
\text { (Lycopersicon } \\
\text { esculentom var. } \\
\text { Roma) }\end{array}$ & MW-HAD & $\begin{array}{l}\mathrm{d}=0.04-0.05 \mathrm{~mm} ; \mathrm{v}=2 \mathrm{~m} / \mathrm{s} \\
\mathrm{RH}=35 \% ; \mathrm{T}=55^{\circ} \mathrm{C} ; \mathrm{P}=300 \mathrm{~W}\end{array}$ & Reduced drying time by $26 \%$ & - & $\begin{array}{l}\text { Askari et al. } \\
(2009)\end{array}$ \\
\hline
\end{tabular}

time of $31 \mathrm{~min}$. Similar reports on the effect of MW-HAD combination on the drying time, kinetics, efficiency and modelling approach of other fruits like apple (red delicious) (Aghilinategh et al., 2015), apple (golden delicious) (Askari, Emam-Djomeh, \& Mousavi, 2009), strawberries (Askari et al., 2009), citrus lemon (Darvishi, Khoshtaghaza, \& Minaei, 2014), moringa olifera (drumstick fruits) (Dev, Geetha, Orsat, Gariépy, \& Raghavan, 2011) and banana (Ganesapillai, Regupathi, \& Murugesan, 2011) have been well documented as shown in Table 1.

In contrast, Kassem et al. (2011) studied on the comparison between MW combined HAD (MW + HAD; HAD + MW) and HAD of Thompson seedless grape. The results of their study show drying time of both MW + HAD and HAD + MW decreased drastically as compared to the HAD. For example, Fig. $4 a-b$ shows the graph of (a)

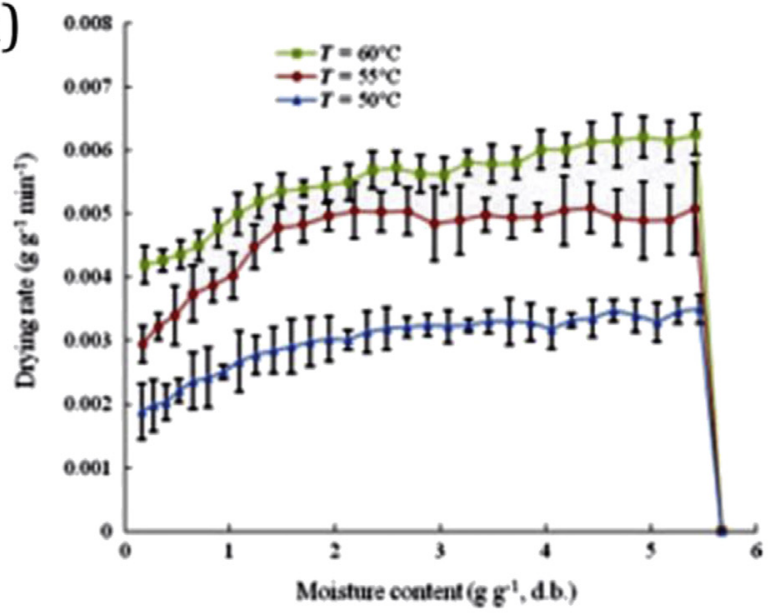

(c)

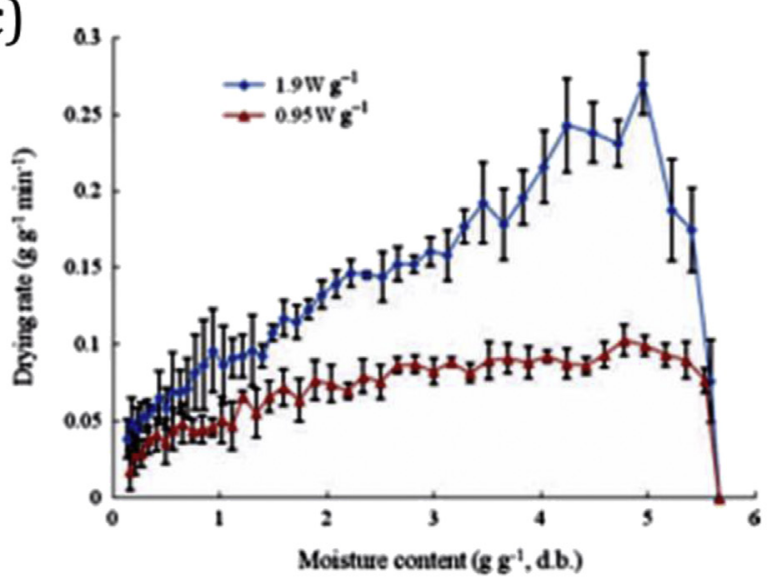

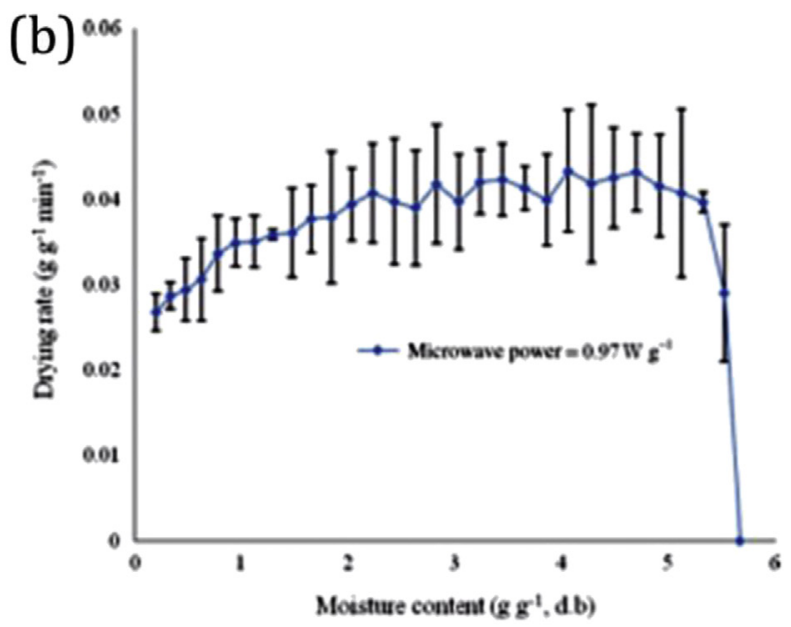

Fig. 3. Drying rate vs moisture content (a) HAD (b) MWD (c) MW-HAD (Adapted from: Sadeghi et al., 2013). 

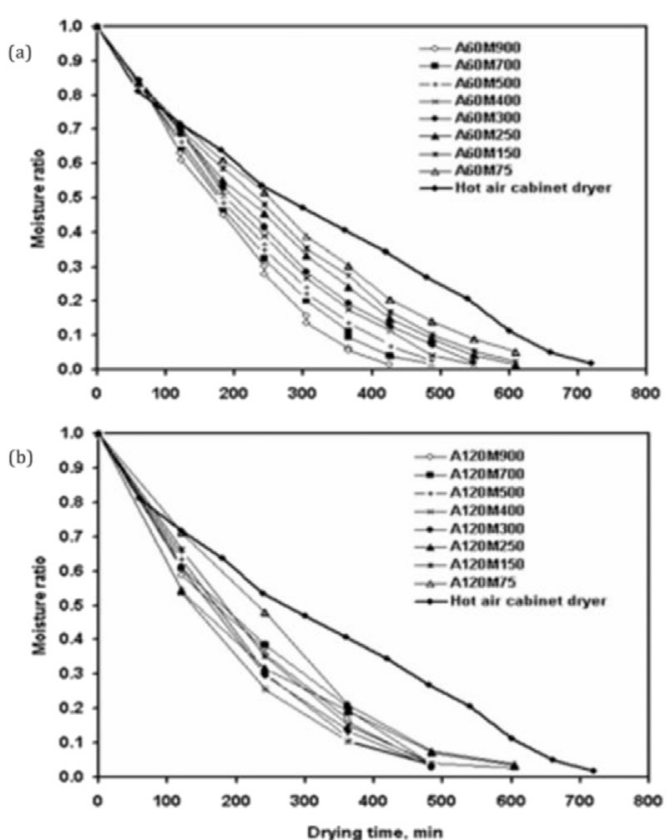
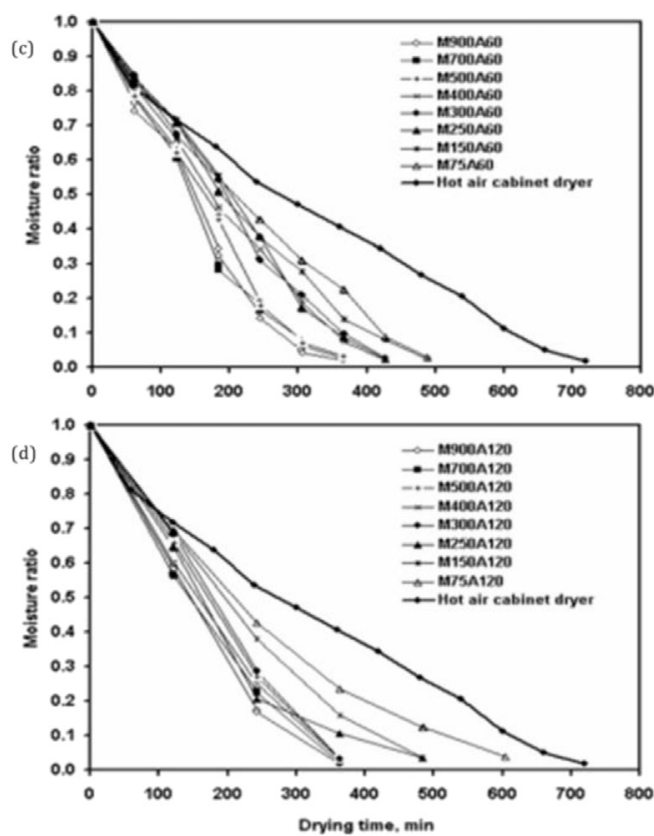

Fig. 4. Thin layer drying curves dried by MW+HAD and HAD+MW as compared to HAD alone (Adapted from: Kassem et al., 2011).

the moisture ratio-time diagram of grapes along the drying period for HAD + MWD combination. From the figure, it can be observed that drying time for the combined method reduced drastically when compared with HAD. Similarly, Fig. $4 c-d$ presents the graph of the moisture ratio-time diagram of grapes along the drying period for MW + HAD drying combination. It was also observed that this method reduced the drying time drastically as compared to HAD alone.

For vegetables, Soysal, Arslan, et al. (2009) reported on the intermittent microwave and hot-air combined drying of oregano. The drying was in two modes namely; IMW-HAD and MW-HAD. The drying process conditions were temperature range of $40-50{ }^{\circ} \mathrm{C}$, MW power of $700 \mathrm{~W}$. On-off timings used in the IMW + HAD were $15 \mathrm{~s}$ on 30 s off, $15 \mathrm{~s}$ on $45 \mathrm{~s}$ off, and $15 \mathrm{~s}$ on $60 \mathrm{~s}$ off. The results of their study show that a higher drying rate (DR) for MW-HAD combination was achieved as compared to HAD. The average drying rate (ADR) was 29.5-59.0 times higher than HAD alone, and 3.5-5.7 times higher than IMW+HAD. This increase can be attributed to the penetration of MW energy into the sample, and the simultaneous heat and mass transfer that take place together with the volumetric heating. More so, MW-HAD had the shortest drying time $(\mathrm{t})$. The drying time when using HAD was 26.5-52.0 times longer than MW-HAD and 4.7-17.3 times longer than IMW+HAD. Specific energy consumption (SEC) for MW-HAD was 12.7-14.0 times lower than HAD, while IMW+HAD was 4.7-11.2 times more efficient than that HAD. In addition, an Increase in PR resulted in a remarkable reduction in SEC. However, MW-HAD resulted in significant decreased in colour parameters when compared to HAD and MWD, respectively. The reduction in the colour values may be due to intense heating as a result of high MWP of $700 \mathrm{~W}$ used during MW-HAD drying.

Soysal, Ayhan, et al. (2009) also applied IMW+HAD and MWHAD combination in the drying of red pepper. From the results of their study, they reported that HAD drying time was 10.4-19.6 times longer than MW-HAD and 2.5-11.8 times longer than IMW + HAD, depending on the MWP level, Pulse ratio (PR), and drying temperature (T). IMW + HAD at $35{ }^{\circ} \mathrm{C}$ with PR of 3.0 at $597.20 \mathrm{~W}$ resulted in considerable reduction in drying time and produced final dried product of high quality with improved colour, textural and sensory attributes. Hemis et al. (2015) also collaborated the effect of MW+HAD on the drying rate of vegetables. They applied MW $+\mathrm{HAD}$ for the drying of canola seed. The drying condition used were temperature range of $40-60{ }^{\circ} \mathrm{C}$ and $\mathrm{MW}$ power range of $0-750 \mathrm{~W}$. The result of their study showed $97 \%$ increase in the drying rate of canola seed under combined MW + HAD when compared to HAD. They identified coupled mathematical model as the most suitable in describing the drying behaviour of canola seed. Such a modelling approach can help identify and regulate overheating.

Horrungsiwat, Therdthai, and Ratphitagsanti (2016) applied MW-HAD in the drying of broccoli stalk. They observed a reduction in drying time by $42-55 \%$ when compared with HAD alone, and the effective diffusivity was twice higher than those of HAD. The best colour retention resulted from the application of MW-HAD at $100 \mathrm{~W}-40^{\circ} \mathrm{C}$, this led to getting colour parameters $\left(\mathrm{L}^{*}, \mathrm{a}^{*}, \mathrm{~b}^{*}\right)$ values of dried product close to those of fresh broccoli stalk. Similarly, several researchers have studied the combination of MW and HAD of other vegetables including beetroot, which resulted in higher water activity, high TSS and improved hardness as compared to fresh samples (Kaur \& Singh, 2014), tomatoes (Askari et al., 2009; Workneh \& Oke, 2013), potatoes (Malafronte et al., 2012) and mushroom (Askari et al., 2009; Wang, Zhang, \& Mujumdar, 2014a), as presented in Table 1.

Modelling is a useful tool for predicting the drying kinetics of agricultural crops to select optimum drying conditions, and to understand the effect of different drying conditions on heat and mass transfer process (Defraeye, 2014; Erbay \& Icier, 2010; Onwude et al., 2016a). Several mathematical models were used by different authors to explain the process of water removal from different agricultural crops during combine MW and HAD (Table 1). The most appropriate models in describing the drying process of agricultural crops using this drying method include Midilli et al. model, Twoterm model, Logarithmic model, Lewis model, Coupled mathematical model, Page model, and Diffusion model. However, collectively, the Midilli et al. and Page models are the most often used in describing the drying kinetics of agricultural crops based on 
studies in scientific literature. These models were applicable in describing the drying kinetics of banana, lemon, hawthorn, moringa orifera, papaya, pumpkin, rice, soybeans, seedless grape, and tomatoes (see Table 1).

Overall, combining MW and hot-air drying could provide up to 92\% reduction in drying time, 97\% increase in the drying rate, reduced shrinkage effect and up to $50 \%$ reduction in the required energy consumption when compared to HAD, depending on the crop to be dried, crop dimension, drying conditions, and the mode of combination. Moreover, the most appropriate combination mode for drying of agricultural crops, based on the above-mentioned performance indices, is the MW-HAD combination mode. The quality of the crops during MW and hot-air drying can be optimized by controlling the temperature of the product and the microwave power, as these 2 parameters have shown to affect the quality of agricultural crops. The best modelling approach for predicting the drying behaviour of agricultural crops during combined MW and HAD drying is the semi-theoretical thin layer models (see Table 1.). In addition, the simultaneous and periodical combination of MWD and HAD parameters (e.g. T and P) during single drying process can increase the overall efficiency of the drying process while maintaining products quality. More so, to further reduce energy demand and avoid risk of overheating, it is possible to apply MW both simultaneously and periodically with hot air in same drying process, such a scheme could lead to a decrease in the drying time up to $68 \%$ and low energy consumption of $3.3 \pm 0.1 \mathrm{kWh}$, in comparison with HAD (Łechtańska et al., 2015).

\subsection{Infrared and hot-air combined drying}

Unlike MWD, which involves generating heat by the application of certain EM fields, IR drying (IRD) is carried out by a different type of EM radiation whose wavelengths ranges from 0.78 to $1000 \mu \mathrm{m}$. IR radiation based on this spectrum is often categorised as near or short-infrared (NIR or SIR: $0.78-1.40 \mu \mathrm{m}$ ), medium-infrared (MIR: 1.4-3.0 $\mu \mathrm{m}$ ), and far-infrared (FIR: 3.0-1000 $\mu \mathrm{m}$ ) (Riadh et al., 2015; Mongpraneet et al., 2004, 2016; Rastogi, 2012a, 2012b). A solid body exposed to radiation of these wavelengths can absorb the thermal energy generated in a thin layer at the surface (Nowak \& Lewicki, 2004). IR radiation can be felt as radiant heat flux, which can be frequently adjusted between NIR-FIR to obtain high drying rate for different agricultural crops.

When IR energy strikes on an agricultural crop, charges in the form of vibrational, rotational and electronic states of atom and molecules are created (Moses et al., 2014; Rastogi, 2012b; Sakai \& Hanzawa, 1994), without necessary heating the surrounding air. These charges depend on the temperature, and emissivity of the emitter. During IR heating, maximum radiation can be attained at a wavelength controlled by temperature level and IR heating elements (Krishnamurthy, Khurana, Jun, Irudayaraj, \& Demirci, 2008). This concept can be further explained by the laws of blackbody radiation such as Wien's displacement law, Stefan-Boltzmann's law, and Planck's law (Krishnamurthy et al., 2008; Rastogi, 2012a).

Moreover, for a successful application of IRD on agricultural crop, the IR radiation properties and that of the particular crop must synchronize (Nowak \& Lewicki, 2004; Ratti \& Mujumdar, 2014). Consequently, several researchers have identified the material's emissivity, reflectivity, absorptivity and transmissivity as important properties of interest (Jaturonglumlert \& Kiatsiriroat, 2010; Nowak \& Lewicki, 2004; Nuthong, Achariyaviriya, Namsanguan, \& Achariyaviriya, 2011; Wang et al., 2014a). Krishnamurthy et al. (2008) also presented a summary of the absorption wavelengths of various relevant component for agricultural crops.

IR technology provides improved efficiency for electrical energy conversion into heat, increased surface heating uniformity, decreased net heating time, increased retention of product quality attributes, significant reduction in energy demand, and simple equipment set-up (El-mesery \& Mwithiga, 2015; Sadin, Chegini, \& Khodadadi, 2014). However, the most common application of IR heating involves combined IR and HAD.

Combined IR and HAD provides a synergistic effect, which often results in reduced drying time, reduced energy consumption, and greater efficiency with a higher heat and mass transfer rate. A schematic view of laboratory scaled combined IR and HA dryer developed by Nuthong et al. (2011) is shown in Fig. 5. During combined IR and HAD, energy emitted by the heating element is transferred to the surface of the product without necessary heating the surrounding air, thereby minimizing loss of energy. Specifically, the mechanism of operation for combined IR and HAD of agricultural crops involves the exposure of crop to IR radiation, this increases the molecular vibration at the inner surface layers of the crop, leading to an increase in the rate of moisture movement from inside the material towards the surface. Water vapour formed at the surface of the material can then be easily removed by convective air, thereby reducing temperature of the product for improved dried product quality (Praveen Kumar et al., 2006; Hebbar and Rastogi, 2001).

Similar to combined MWD and HAD, combination of IR and HAD has recently received much attention as novel thermal heat drying method, either to augment or completely replace the conventional drying technology to improve overall process efficiency. Several authors have based the advantage of this technology over others on the synergistic effect and mechanism of operation, which result in reduced drying time and better quality of dried products. A list of recent publications in the past 9 years is presented in Table 2 . The table also summarizes the various significant findings and combination modes, which include: simultaneous IR and HAD (IR-HAD), and sequential IR and HAD (HAD + IRD; IR + HAD) drying of agricultural crops. More so, some studies expressed IR in terms of NIR, MIR and FIR, as earlier explained.

\subsubsection{Application to agricultural crops}

3.2.1.1. Cereals. Tuncel, Yilmaz, Kocabiyik, Öztürk, and Tunçel (2010) studied the IR-HAD combination of corn. The results of their study showed that IR-HAD combination has a positive impact on the drying time of the entire process. The performance evaluation of their study indicated a $60.7 \%$ decrease in the drying time when compared to drying using HAD, and 28.6\% decrease when compared with IR alone. The reduction in the drying time may be due to rapid diffusion of water within the product to the surface due to IR energy and simultaneous removal of water vapour from the surface to the atmosphere by convective air. This short drying duration led to lower specific energy consumption (SEC) and higher amount of carotenoid content of corn as compared to experiments using HAD or IR alone, respectively.

In a similar manner, Zare, Naderi, and Ranjbaran (2015) argued that drying using IR-HAD combination results in low SEC, higher drying rate, and shorter drying time at constant intensity and velocity when compared with HAD drying. This method did not significantly affect the quality of paddy when compared with drying using HAD. A different combination mode was used in the drying of parboiled rice. The sequential HAD + IRD combination was adopted as reported by Bualuang, Tirawanichakul, and Tirawanichakul (2013). They observed a shortened drying time due to higher heat and mass transfer coefficient, increase in the rate of diffusion as the IR intensity increases, and higher head rice yield compared to HAD and IR, respectively. They also noticed that the yellowness and whiteness were significantly affected by combined HAD + IRD. 


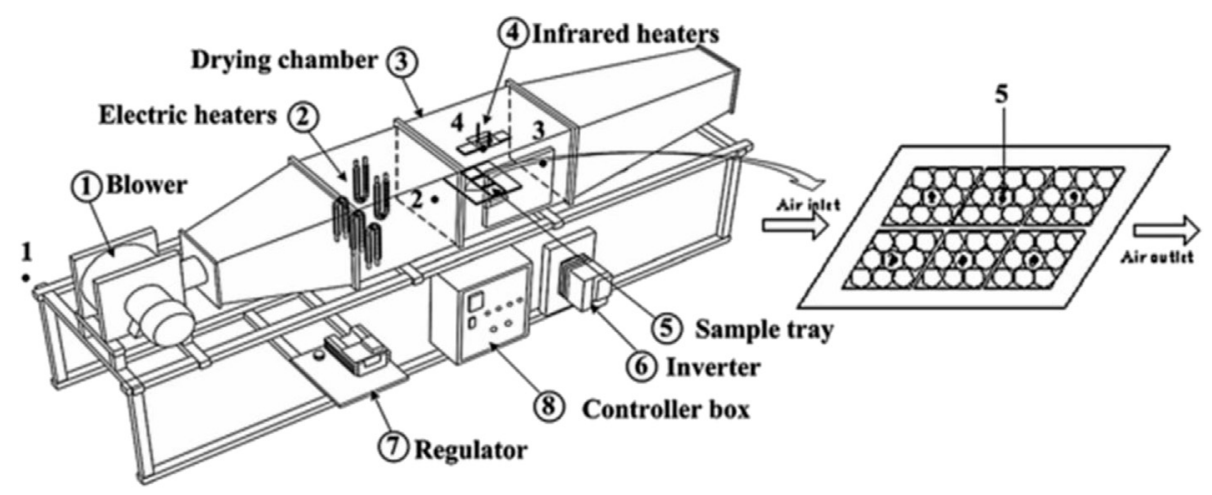

Fig. 5. A typical laboratory scale combined IR and HA dryer (Adapted from: Nuthong et al., 2011).

3.2.1.2. Fruits and vegetables. El-mesery and Mwithiga (2015) investigated the performance of combined IR - HAD of apples. They observed a higher drying rate $(7.40 \mathrm{~g} / \mathrm{g}$ of dry matter per min) when compared with HAD (4.16 g/g of dry matter per min). In addition, there was $57.5 \%$ reduction in drying time as compared to drying using HAD. The SEC of HAD was $488 \%$ higher than that using IR-HAD. The dryer efficiency was reported to be $57.76 \%$ using combined IR-HAD while that of HAD was $10.8 \%$. They also investigated the effect of drying on the colour of apple. It was noticed that the total colour difference of apples dried using IR-HAD was lower compared to those dried using HAD. This is because of the shorter drying time required to when using the combined mode as compared to longer drying time required during HAD.

In a separate study, Ponkham, Meeso, Soponronnarit, and Siriamornpun (2012) observed that the reduced drying time during IR-HA combined drying of pineapple is as a result of an increase in IR intensity which increases the rate of moisture removal and product temperature, leading to higher heat and mass transfer and lesser drying time. This observation was collaborated by Chen et al. (2015) in the combined short-medium IR and HAD of jujube fruit. They also reported a $33-83 \%$ reduction in the total drying time of $\mathrm{HAD}$, when using the combined method. The drying rate values using SMIR-HAD were 2 times higher than those of HAD $\left(80-90{ }^{\circ} \mathrm{C}\right)$. The higher drying rate maybe due to higher heating efficiency as a result of IR intensity.

Conversely, Nuthong et al. (2011) argued that the reduced drying time during combined IR-HAD is due to the combined effect of temperature and IR power. However, Nathakaranakule, Jaiboon, and Soponronnarit (2010) observed that the effect of IRP at higher temperatures above $60{ }^{\circ} \mathrm{C}$ becomes less significant during the drying of Longan. Jaturonglumlert and Kiatsiriroat (2010) also studied on the combined Far-IR and HAD of Longan. They observed that reduced drying time was due to increased heat flux from $1.9 \mathrm{~kW} / \mathrm{m}^{2}$ (HAD) to $4.20 \mathrm{~kW} / \mathrm{m}^{2}$.

Motevali et al. (2011) demonstrated that combined IR-HAD can be used as an alternative to conventional methods for postharvest drying of vegetables such as mushroom. IR-HAD achieved a decreased drying time with increasing IR intensity. The drying time was shorter when compared to HAD. Wang et al. (2014a) attributed the short drying time ( $55 \%$ reduction of HAD) during the combined MIR-HAD of mushroom to the rapid heating of the crop by IR energy, which intensifies the rate of moisture migration towards the surface of the product, while the convective air flow ensures the simultaneous moisture evaporation from the surface to the atmosphere, thereby reducing the total drying time and energy requirement. This is not the case when drying using only HAD. They also reported that MIR-HAD gave a better-uniformed product than $\mathrm{HAD}$, with minimal shrinkage and lower hardness upon rehydration. Similar findings have been reported by Mihindukulasuriya and Jayasuriya (2015). They observed a 60\% reduction in drying time when compared with HAD alone during the IR-HAD of ripe chilli. However, the quality characteristics, in terms of capsaicin content and colour were similar to those dried using HAD alone.

Sadin et al. (2014) described the effect of IRP in the combined IRHAD of tomatoes. Using IR power of $1.5 \mathrm{~kW}$, and distance between IR emitter and the sample, $d_{e}(10-70 \mathrm{~cm})$, they observed that drying rate is directly proportional to IR Power. They also demonstrated that an increase in $d_{e}$ increases drying time due to the reduction of heat transfer to the product. In the same vein, Vishwanathan, Hebbar, and Raghavarao (2010) studied the combined IR-HAD of carrot. They observed a reduced drying time of about $48 \%$ when compared to results of HAD. Carotenoid retention was also higher by $14 \%$ as compared to HAD.

On the other hand, Łechtańska et al. (2015) demonstrated the sequential combined IR and HAD of green pepper. They observed about $38 \pm 2.7 \%$ reduction in drying time when compared to HAD. Rapid increase in the drying rate only at the beginning of the drying process, due to the application of only IR, was also observed. The increased drying rate, only at the start of the drying process can be explained by the wetness of the material before the commencement of the drying process, thus the rate of moisture removal from the surface to the atmosphere increases. Later, when the surface moisture reduces and more moisture concentrate towards the interior of the crop, the influence of IR on the crop decreases due to its surface action and weak penetration into the material. Hence, the introduction of HA. This process is done periodically until safe moisture is attained. The drawback of using this combination mode can be seen in the increase in energy consumption from $6.5 \pm 0.1 \mathrm{kWh}$ to $6.9 \pm 0.1 \mathrm{kWh}$, and a low vitamin $\mathrm{C}$ retention (16.86\%) when compared to HAD (46.54\%).

In terms of modelling approach, the most appropriate models in describing the drying process of agricultural crops using combined IR and HAD included Page, Two-term, Logarithm and Gab models. These models were applicable in describing the drying kinetics of dill, jujube and rice (see Table 1 ).

Collectively, the increase in drying efficiency and the significant reduction in drying time using the combined IR and HAD could be attributed to the synergistic effect, and the combined effect of IR power, intensity and air temperature. During combined IR - HAD method, the heat transfer is achieved by simultaneous convection and radiation process thereby accelerating the entire drying process. In addition, the simultaneous IR - HAD combination mode proved to be more effective than the sequential approach which results in increased energy demand when compared with IR HAD. The simultaneous combination of IR - HAD resulted in a 
Table 2

Published data on combined infrared and hot-air drying of agricultural crops in the past 9 years.

\begin{tabular}{|c|c|c|c|c|c|}
\hline Agricultural crops & $\begin{array}{l}\text { Combination } \\
\text { mode }\end{array}$ & Drying process conditions & Significant findings & $\begin{array}{l}\text { Modelling } \\
\text { approach }\end{array}$ & References \\
\hline $\begin{array}{l}\text { Apples (Golden } \\
\text { delicious cv.) }\end{array}$ & IR-HAD & $\begin{array}{l}\mathrm{h}=5 \pm 1 \mathrm{~mm} ; \mathrm{IRI}=2000 \mathrm{~W} / \\
\mathrm{m}^{2} ; \mathrm{T}=60^{\circ} \mathrm{C} ; \mathrm{v}=0.6 \mathrm{~m} / \mathrm{s}\end{array}$ & $\begin{array}{l}57.5 \% \text { reduction in drying time; SEC of HAD } \\
\text { was } 488 \% \text { higher than that of IR-HAD; Dryer } \\
\text { efficiency using IR-HAD }=57.76 \% \text { while } \\
\text { HAD }=10.8 \% ; \triangle E \text { was shorter compared to } \\
\text { that of } \mathrm{HAD} \text {. }\end{array}$ & - & $\begin{array}{l}\text { El-mesery and } \\
\text { Mwithiga (2015) }\end{array}$ \\
\hline $\begin{array}{l}\text { Carrot (Daucus carota } \\
\text { L.) }\end{array}$ & IR - HAD & $\begin{array}{l}\mathrm{d}=25 \mathrm{~mm} ; \mathrm{h}=5 \mathrm{~mm} \\
\mathrm{v}=1.4 \mathrm{~m} / \mathrm{s} ; \mathrm{T}=80^{\circ} \mathrm{C} ; \lambda=2.4 \\
-3.0 \mu \mathrm{m}\end{array}$ & $\begin{array}{l}\mathrm{RR}=11.4 \% \text {; Carotenoid retention was } \\
\text { higher by } 14 \% \text { as compared to HAD; BI value } \\
(100.53) \text { compared to HAD ( } 107.49) \\
D_{\text {eff }}=4.81 \times 10^{-8} \text { as compared to } \\
2.28 \times 10^{-8}\end{array}$ & - & $\begin{array}{l}\text { Vishwanathan } \\
\text { et al. (2010) }\end{array}$ \\
\hline Chilli (Pickino) & IR-HAD & $\mathrm{T}=50-70{ }^{\circ} \mathrm{C} ; \lambda=2.4-3.0 \mu \mathrm{m}$ & $\begin{array}{l}\text { HAD consumed } 33.5 \% \text { more power than IR- } \\
\text { HAD }\end{array}$ & & $\begin{array}{l}\text { Mihindukulasuriya and } \\
\text { Jayasuriya (2015) }\end{array}$ \\
\hline Corn (Zea mays) & IR - HAD & $\begin{array}{l}\mathrm{v}=1 \mathrm{~m} / \mathrm{s} ; \mathrm{T}=45^{\circ} \mathrm{C} \\
\mathrm{IRP}=0.8 \mathrm{~kW} ; d_{e}=90 \mathrm{~mm}\end{array}$ & $\begin{array}{l}\text { Lower SEC value compared with HAD; } \\
\text { Higher carotenoid content compared to } \\
\text { HAD and IR alone, respectively. }\end{array}$ & - & Tuncel et al. (2010) \\
\hline $\begin{array}{l}\text { Dill (Anethum } \\
\quad \text { graveolens L) greens }\end{array}$ & MIR-HAD & $\begin{array}{l}\mathrm{v}=1.3 \mathrm{~m} / \mathrm{s} ; \mathrm{T}=50 \pm 2{ }^{\circ} \mathrm{C} \\
\mathrm{RH}=50-60 \%\end{array}$ & - & $\begin{array}{l}\text { Semi theoretical } \\
\text { Page model }\end{array}$ & $\begin{array}{l}\text { Madhava Naidu } \\
\text { et al. (2015) }\end{array}$ \\
\hline $\begin{array}{l}\text { Green pepper } \\
\text { (Capsicum annuum } \\
\text { L.) }\end{array}$ & $\begin{array}{l}\mathrm{IR}+\mathrm{HAD} \\
\text { (periodically) }\end{array}$ & $\begin{array}{l}\mathrm{L}=3 \mathrm{~cm} ; \mathrm{W}=2 \mathrm{~cm} ; \\
\mathrm{V}=1.8 \pm 0.1 \mathrm{~m} / \mathrm{s} ; \mathrm{T}=75^{\circ} \mathrm{C} ; \\
\mathrm{IRP}<240 \mathrm{~W} \text { depending on } \\
\text { temp and } \mathrm{MC}\end{array}$ & $\begin{array}{l}\text { Increased energy consumption from } \\
6.5 \pm 0.1 \mathrm{kWh} \text { to } 6.9 \pm 0.1 \mathrm{kWh} \text {; lowest } \\
\text { value of VC retention ( } 16.86 \%) \text { due to high } \\
\text { temperature of } 75^{\circ} \mathrm{C} \text { as compared to } 46.54 \% \\
\text { when using HAD. }\end{array}$ & - & $\begin{array}{l}\text { Łechtańska } \\
\text { et al. (2015) }\end{array}$ \\
\hline $\begin{array}{l}\text { Jujube (Zizyphus jujube } \\
\quad \text { cv. zhanhuadongzao) }\end{array}$ & SMIR-HAD & $\begin{array}{l}\mathrm{v}=2.11 \mathrm{~m} / \mathrm{s} ; \mathrm{IRP}=1125 \mathrm{~W} \\
d_{e}=11 \mathrm{~cm} ; \mathrm{T}=60-90^{\circ} \mathrm{C} ; \lambda=1 \\
-4 \mu \mathrm{m}\end{array}$ & $\begin{array}{l}D_{\text {eff }} \text { was } 2 \text { times higher than HAD ranging } \\
\text { from } 9.01 \times 10^{-8}-2.28 \times 10^{-7} \mathrm{~m}^{2} / \mathrm{s}\end{array}$ & $\begin{array}{l}\text { Semi theoretical } \\
\text { Two terms model; } \\
\text { Logarithm } \\
\text { model }\end{array}$ & Chen et al. (2015) \\
\hline Longan & FIR - HAD & $\begin{array}{l}d_{1}=15 \mathrm{~mm} ; d_{2}=25 \mathrm{~mm} \\
\mathrm{~T}=65^{\circ} \mathrm{C} ; \mathrm{RH}=10-90 \% ; \\
\mathrm{IRP}=250-450 \mathrm{~W} ; \mathrm{IRI}=0.6 \\
-1.2 \mathrm{Wcm}^{-2} ; \lambda=7-1000 \mu \mathrm{m}\end{array}$ & $\begin{array}{l}\text { FIR had less effect at higher drying } \\
\text { temperature }\end{array}$ & - & $\begin{array}{l}\text { Nathakaranakule } \\
\text { et al. (2010) }\end{array}$ \\
\hline $\begin{array}{l}\text { Longan (Dimocarpus } \\
\text { longan lour) }\end{array}$ & FIR - HAD & $\begin{array}{l}\mathrm{v}=0.5 \mathrm{~m} / \mathrm{s} ; \mathrm{T}=300-500{ }^{\circ} \mathrm{C} \\
d_{e}=10-30 \mathrm{~cm} ; \mathrm{IRP}=800 \mathrm{~W} \\
\text { (maximum) }\end{array}$ & $\begin{array}{l}\text { shorter drying time due to increased heat } \\
\text { flux from } 1.9 \mathrm{~kW} / \mathrm{m}^{2} \text { (HAD) to } 4.20 \mathrm{~kW} / \mathrm{m}^{2}\end{array}$ & - & $\begin{array}{l}\text { Jaturonglumlert } \\
\text { and Kiatsiriroat (2010) }\end{array}$ \\
\hline Longan (Edor) & IR - HAD & $\begin{array}{l}\mathrm{d}=25-30 \mathrm{~mm} ; \mathrm{IRP}=300 \\
-700 \mathrm{~W} ; \mathrm{T}=40-80^{\circ} \mathrm{C} ; \mathrm{v}=0.5 \\
-1.5 \mathrm{~m} / \mathrm{s}\end{array}$ & $\begin{array}{l}\text { Higher air temperature and IRP led to high } \\
\text { drying rates. }\end{array}$ & - & Nuthong et al. (2011) \\
\hline Mushroom & IR-HAD & $\begin{array}{l}\mathrm{v}=5 \mathrm{~m} / \mathrm{s} ; \mathrm{RH}=30 \pm 2 \% \\
\mathrm{IRI}=0.49-0.22 \mathrm{~W} / \mathrm{cm}^{2} ; \mathrm{T}=40 \\
-60{ }^{\circ} \mathrm{C}\end{array}$ & $\begin{array}{l}\text { Drying time decreased with increasing IR } \\
\text { intensity }\end{array}$ & - & $\begin{array}{l}\text { Motevali, Minaei, } \\
\text { Khoshtaghaza, and } \\
\text { Amirnejat (2011) }\end{array}$ \\
\hline $\begin{array}{l}\text { Mushrooms (Lentinus } \\
\text { edodes) }\end{array}$ & MIR-HAD & $\begin{array}{l}\lambda=2.4-3.0 \mathrm{~mm} ; \mathrm{IRP}=400 \mathrm{~W} \\
d_{e}=14 \mathrm{~cm} ; \mathrm{T}=60^{\circ} \mathrm{C} \\
\mathrm{v}=1.0 \mathrm{~m} / \mathrm{s}\end{array}$ & $\begin{array}{l}\text { MIR-HAD gave a better uniformed product } \\
\text { than HAD; minimal shrinkage was } \\
\text { observed; lower hardness upon rehydration }\end{array}$ & - & Wang et al. (2014a,b) \\
\hline Paddy & IR - HAD & $\begin{array}{l}\mathrm{v}=0.10-0.2 \mathrm{~m} / \mathrm{s} ; \mathrm{T}=30 \\
-50^{\circ} \mathrm{C} ; \mathrm{IRI}=2000-6000 \mathrm{~W} / \mathrm{m}^{2}\end{array}$ & $\begin{array}{l}\text { At lower IRI, percentage cracked paddy was } \\
\text { lower compared with HAD at same } \\
\text { temperature; Best condition is } 30^{\circ} \mathrm{C} \text {, } \\
0.15 \mathrm{~m} / \mathrm{s} \text { and } 2000 \mathrm{~W} / \mathrm{m}^{2}\end{array}$ & - & Zare et al. (2015) \\
\hline Pineapples & FIR - HAD & $\begin{array}{l}\mathrm{h}=15 \mathrm{~mm} ; d_{1}=3.0 \mathrm{~cm} ; \\
d_{2}=9.0 \mathrm{~cm} ; \mathrm{v}=0.5-1.5 \mathrm{~m} / \mathrm{s} ; \\
\mathrm{IRI}=1-5 \mathrm{~kW} / \mathrm{m}^{2} ; \mathrm{T}=40-60{ }^{\circ} \mathrm{C}\end{array}$ & $\begin{array}{l}\text { Increased in intensity increased moisture } \\
\text { removal rate and product temperature }\end{array}$ & - & Ponkham et al. (2012) \\
\hline $\begin{array}{l}\text { Potatoes (Solanum } \\
\text { tuberosum L.) }\end{array}$ & IR - HAD & $\begin{array}{l}\mathrm{L}=17 \mathrm{~mm} ; \mathrm{W}=17 \mathrm{~mm} \\
\mathrm{~h}=5 \mathrm{~mm} ; \mathrm{v}=1.4 \mathrm{~m} / \mathrm{s} \\
\mathrm{T}=80^{\circ} \mathrm{C} ; \lambda=2.4-3.0 \mu \mathrm{m}\end{array}$ & $\begin{array}{l}\text { RR } 12.5 \% \text {; Lower BI value }(32.48 \pm 0.44) \\
\text { compared to } \mathrm{HAD}(42.53 \pm 1.11) \\
D_{\text {eff }}=3.65 \times 10^{-8} \text { as compared to } \\
1.37 \times 10^{-8}\end{array}$ & - & Vishwanathan et al. (2010) \\
\hline Rice (Perboiled) & $\begin{array}{l}\mathrm{HA}+\mathrm{IRD} \\
\text { (sequential) }\end{array}$ & $\begin{array}{l}\mathrm{v}=1 \pm 0.2 \mathrm{~m} / \mathrm{s} ; \mathrm{T}=60-100^{\circ} \mathrm{C} \\
\mathrm{IRP}=1000-1500 \mathrm{~W} ; \mathrm{RH}=11 \\
-87 \%\end{array}$ & $\begin{array}{l}D_{\text {eff }} \text { increased with increased IRI; Higher } \\
\text { HRY compared to IR alone; Yellowness and } \\
\text { whiteness were significantly affected. }\end{array}$ & GAB model & Bualuang et al. (2013) \\
\hline Tomatoes & IR- HAD & $\begin{array}{l}\mathrm{h}=5 \mathrm{~mm} ; \mathrm{v}=0.6-1.1 \mathrm{~m} / \mathrm{s} \\
\mathrm{T}=60-80{ }^{\circ} \mathrm{C} ; \lambda=3-4.2 \mu \mathrm{m} \\
\mathrm{IRP}=1.5 \mathrm{~kW} ; d_{e}=10-70 \mathrm{~cm}\end{array}$ & $\begin{array}{l}\text { Drying rate is directly proportional to IRP; } \\
\text { increased } d_{e} \text { increases drying time due to } \\
\text { reduction of heat transfer to the product }\end{array}$ & - & Sadin et al. (2014) \\
\hline
\end{tabular}

reduction in the drying time of up to $60 \%$ (cereals) and up to $83 \%$ (fruits and vegetables) depending on IR power when compared with drying using HAD. Also, the efficiency of this process was improved (58\% of HAD). The specific energy consumption required during HAD of agricultural crops can be up to $488 \%$ higher than combined IR - HAD. Furthermore, combined IR and HAD method provided final products with great quality. Combined IR-HAD of agricultural crops was found to be dependent on the drying conditions used, the power level, the combination mode and the type of the crop. Thus, there is a need to determine the optimum drying conditions when applying this drying method on agricultural crops.

\subsection{Radio-frequency and hot-air combined drying}

Radio frequency (RF) waves are the EM radiations with frequencies between 1 and $300 \mathrm{MHz}$ (Shinde, Das, \& Datta, 2013). In radio frequency applications of agricultural crops, such as drying, the mechanism of operation involves generation of heat as a result of the interaction between EM field and polarized molecules in the crops which are either of bipolar or ionic nature. The product is 
placed between two electrodes where the alternating energy from electric field generated by RF generator, results in polarization. The resulting alternation of the electric field causes the polar molecule in the product to likewise alternate. This leads to friction which creates heat energy throughout the product (Marra, Zhang, \& Lyng, 2009; Piyasena, Dussault, Koutchma, Ramaswamy, \& Awuah, 2003).

RF drying (RFD) method has been seed to provide better penetration depth, enhanced heating uniformity and more stable product temperature (Marra et al., 2009; Wang et al., 2011). Several authors have outlined the early applications of RF for drying of wood (Balakrishnan, Vedaraman, Sundar \& Muralidharan 2004), paper (Balakrishnan et al., 2004), textiles (Balakrishnan et al., 2004), bakery (Chou \& Chua, 2001), and pharmaceutical products (Piyasena et al., 2003). However, apart from Murphy, Morrow, and Besley (1992), most of these applications, which involve nonagricultural crops such as wood, were carried out extensively either using RF drying alone or a combination of RF-vacuum (Dziak, 2008; Koumoutsakos, Avramidis, \& Hatzikiriakos, 2001; Li \& Lee, 2008).

Evident suggest that the most successful application often combine two or more technologies (RF-HA, RF-HP, etc.). While the application of combined MW-HAD and IR-HAD in the drying of agricultural crops is well established to some extent, the use of combined RF and HAD of agricultural crops has not been well researched on in the recent years, despite the enormous advantages associated with using this technique.

Wang et al. (2014a) studied the drying of mushrooms using combined HA-RF drying. They observed a $46.7 \%$ reduction in the drying time when compared with drying using HAD. The drying efficiency was higher than that of HAD, due to the variations in the modes of energy transmission of the different drying methods. In a similar manner, Wang et al. (2014b) studied the drying of Macadamia nuts using combined RF-HAD system (Fig. 6). They demonstrated that during RF-HAD, an initial rapid increase in the drying time was observed and slowed thereafter. The drying curve generally followed a typical exponential decay, showing an internal mass transfer inside the material. The results of the quality study showed that while the overall quality was reduced, the values were still within limits acceptable to the nut industry.

Shinde et al. (2013) examined the performance of hybrid $\mathrm{HA}+\mathrm{RF}$ dryer (HA + RFD) during the sequential drying of tea leaves, using power emission between 20 and $16 \mathrm{~kW}$. They observed that RF penetration has significant effect on the drying rate and quality attributes of different leaves. There was reduced drying time when compared with HAD. They also stated that the nature and condition of the material to be dried affects the drying rate. It was also stated that tea leaves were macerated, leading to reduced size, and dried faster than others. The energy consumption using combined HA + RFD were the lowest when compared with HAD and RF alone, with only $40 \%$ of the HAD energy demand.

In summary, the energy consumption using this method is lower compared to that of conventional HAD, and can be up to $40 \%$ of the HAD energy demand. It was also observed that the nature and condition of the material to be dried affects the drying rate.

\section{Conclusion}

Recent advances in drying technology have seen enormous amount of interest on hybrid or hurdle (combined) technology as a future potentially effective and viable method for drying of agricultural crops, largely to increase overall process efficiency, by reducing processing time and energy, and preserving the important quality attributes of agricultural crops. In this sense, the use of novel thermal technologies in combination with hot-air drying presents several potential benefits to food, and agricultural preservation and processes. The recent application of novel thermal technologies (MW, IR, and RF) combined with hot-air drying of agricultural crops, specifically, cereals, fruits, vegetables, and oil seeds, are reported and summarized in this paper.

The scientific literature reviewed have shown that combined MW and HAD provides distinctive opportunities in the development of advanced agricultural crops drying technologies. The most significant advantage of combining MW and HAD is the substantial reduction in the drying time and energy consumption as well as, increase in the drying rate and overall efficiency. However, mechanism of heating, and quality of the final dried products using this method still presents a great challenge that needs further investigation. Similarly, the application of IR and HAD drying of agricultural crops has been shown to be a promising novel technology due to its penetration speed and mechanism of heating, reduction in drying time and energy consumption, and improvement in the quality of dried products, however its penetrating powers and depth are still limited. This investigation has further revealed that the combination of RF and HAD has great potential in drying of agricultural crops.

In regards to modelling of agricultural crops using these technologies, the most often used models are the semi-theoretical and empirical models. However, different models were seen to describe the drying process of different crops. For combined IR and HAD, less than $18 \%$ of researchers modelled the drying process of agricultural crops using this method. This knowledge gap could be a basis for future research domain. Therefore, for effective optimization of existing systems, and design of new concepts, further investigation is required. A modelling approach that will minimize or eliminate experimental trials, and can be applied to a wide range of crops, irrespective of the mode of heat transfer is indispensable.

In terms of cost and practical difficulties in adopting these

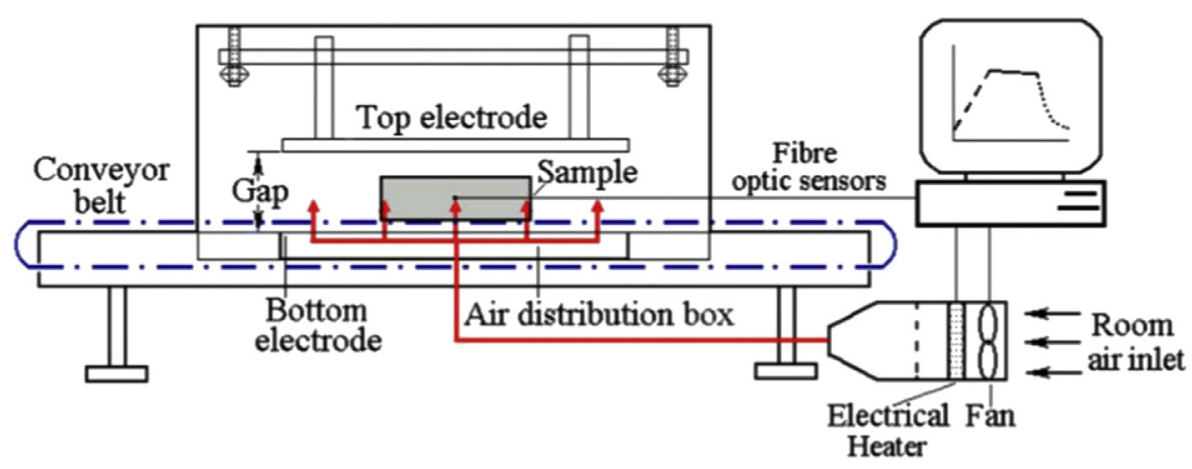

Fig. 6. A typical schematic view of a pilot-scale combine RF-HA dryer (Adapted from: Wang et al., 2010). 
technologies, the MW and RF are the most expensive to set up and difficult to adapt to HA dryers. More so, for combined RF and HAD, the configuration and optimization of the dryer parameters is very difficult due to the RF mode of transmission. In addition, RF combined HAD requires advance knowledge of the electrical properties of agricultural crop to be dried, adding to the positioning of the electrodes which have to be in contact with the crop during drying. This makes the whole drying process to be expensive and cumbersome. Furthermore, it is quite difficult to recover heat/energy during combined MW and HAD or combined RF and HAD, when compared to IR and HAD or HAD alone. This is as a result of high MW and RF power needed during the drying processes. All, these are some of the limitation associated with adopting this technology on an industrial scale. However, the advantages of using these methods are enormous thus, further studies on the design and optimization of combined MW and HAD, and RF and HAD systems are encouraged. Optimization can be carried out by the use of generic algorithm to determine the best process conditions, operation parameters, and energy/exergy requirements. Embedded simulation software, and automated thermographic system can be developed to help monitor the entire drying process, making the process less cumbersome thereby increasing the overall drying process efficiency. On the other hand, the combination of IR and HAD presents greater potential due to its affordability and adaptability.

In general, the combination of MW and HAD, and IR and HAD were adequate in increasing the overall drying process efficiency of cereals, oilseeds, fruits and vegetables when compared with HAD alone. On the other hand, the combination of RF and HAD was found suitable for enhancing the drying process of only vegetables. In addition, while the use of MW and IR combined HAD have been established to some extent, even in the industry, the application of $\mathrm{RF}$ combined HAD must continue to be developed and extensively researched on, as it provides great potential for advance agricultural dried crops. Moreover, the variations in the results (e.g. drying time, efficiency, drying rate and energy consumption) of various agricultural crops may be attributed to differences in drying equipment (Non-standardized laboratory scaled set-up) and drying conditions, these variations and the development of commercial scale hybrid dryers could also be basis for future research.

In conclusion, there are numerous amount of research on drying time, drying rate, and energy consumption as shown in this study. However, there are few research on the quality attributes of agricultural crops during combined novel thermal and HAD. This gap in knowledge could be a serious setback in the commercialization and industrialization of most combined novel thermal and HAD technology, hence a basis for future research. Nonetheless, in terms of product quality, uniformity, drying characteristics, and practical adaptability, combined IR and HAD (IR - HAD) dried agricultural crops resulted in better drying efficiency. Thus, taking into account the above, and cost of drying operation, IR-HAD can be considered as the best combined novel thermal and HAD method for agricultural crops. For increased overall process efficiency, combination of IR-HAD with MW or other novel-thermal technology (e.g RF or inductive heating) would be of great potential. Due to the increased synergistic effect, this combination method could achieve greater reduction in drying time, optimum energy usage, premium product quality and efficient industrial applicability.

\section{References}

Afzal, T. M., Abe, T., \& Hikida, Y. (1999). Energy and quality aspects during combined FIR-convection drying of barley. Journal of Food Engineering, 42(4), 177-182.

Aghilinategh, N., Rafiee, S., Gholikhani, A., Hosseinpur, S., Omid, M., Mohtasebi, S. S., et al. (2015). A comparative study of dried apple using hot air, intermittent and continuous microwave: Evaluation of kinetic parameters and physicochemical quality attributes. Food Science \& Nutrition, 3(6), 519-526.

Akpinar, E. K. (2006). Mathematical modelling of thin layer drying process under open sun of some aromatic plants. Journal of Food Engineering, 77, 864-870.

Alibas, I. (2007). Microwave, air and combined microwave-air-drying parameters of pumpkin slices. LWT - Food Science and Technology, 40(8), 1445-1451.

Amiri Chayjan, R., Kaveh, M., \& Khayati, S. (2014). Modeling drying characteristics of hawthorn fruit under microwave-convective conditions. Journal of Food Processing and Preservation, 39, 239-253.

Andrés, A., Fito, P.. Heredia, A., \& Rosa, E. M. (2007). Combined drying technologies for development of high-quality shelf-stable mango products. Drying Technology, 25(11), 1857-1866.

Arslan, D., \& Ozcan, M. M. (2011). Dehydration of red bell-pepper (Capsicum annuum L.): Change in drying behavior, colour and antioxidant content. Food and Bioproducts Processing, 89(4), 504-513.

Askari, G. R., Emam-Djomeh, Z., \& Mousavi, S. M. (2009). An investigation of the effects of drying methods and conditions on drying characteristics and quality attributes of agricultural products during hot air and hot air/microwaveassisted dehydration. Drying Technology, 27(7-8), 831-841.

Aziz, M., Fushimi, C., Kansha, Y., Mochidzuki, K., Kaneko, S., Tsutsumi, a., et al. (2011). Innovative energy-efficient biomass drying based on self-heat recuperation technology. Chemical Engineering \& Technology, 34(7), 1095-1103.

Balakrishnan, P. A., Vedaraman, N., Sundar, V. J., \& Muralidharan, C. (2004). Radio frequency heating - a prospective leather drying system for future radio. Drying Technology, 22(6), 1969-1982.

Bualuang, O., Tirawanichakul, Y., \& Tirawanichakul, S. (2013). Comparative study between hot air and infrared drying of parboiled rice: Kinetics and qualities aspects. Journal of Food Processing and Preservation, 37(6), 1119-1132.

Chen, Q., Bi, J., Wu, X., Yi, J., Zhou, L., \& Zhou, Y. (2015). Drying kinetics and quality attributes of jujube (Zizyphus jujuba Miller) slices dried by hot-air and shortand medium-wave infrared radiation. LWT - Food Science and Technology, 64(2), 759-766.

Chen, G. (2015). Drying Operations: Agricultural and forestry products. In B. Capehart (Ed.), Encyclopedia of energy engineering and technology (2nd ed. Vol. 1). London, UK: Taylor \& Francis Books. ISBN 978-0-8493-3653-9.

Chong, C. H., Figiel, A., Law, C. L., \& Wojdyło, A. (2013). Combined drying of apple cubes by using of heat pump, vacuum-microwave, and intermittent techniques. Food and Bioprocess Technology, 7(4), 975-989.

Chou, S. K., \& Chua, K. J. (2001). New hybrid drying technologies for heat sensitive foodstuffs. Trends in Food Science and Technology, 12(10), 359-369.

Chua, K. J., Mujumdar, A. S., Chou, S. K., Hawlader, M. N., \& Ho, J. C. (2000). Convective drying of banana, guava and potato pieces: Effect of cyclical variations of air temperature on drying kinetics and color change. Drying Technology, 18(4-5), 907-936.

Ciurzyńska, A., \& Lenart, A. (2011). Freeze-drying - Application in food processing and biotechnology - A review. Polish Journal of Food and Nutrition Sciences, 61(3), 165-171.

Cullen, P. J., Tiwari, B. K., \& Valdramidis, V. P. (2012). Status and trends of novel thermal and non-thermal technologies for fluid foods. In Novel thermal and nonthermal technologies for fluid foods (pp. 1-6). San Diego: Academic Press.

Darvishi, H., Khoshtaghaza, M. H., \& Minaei, S. (2014). Drying kinetics and colour change of lemon slices. International Agrophysics, 28(1), 1-6.

Datta, A. K., \& Ni, H. (2002). Infrared and hot-air-assisted microwave heating of foods for control of surface moisture. Journal of Food Engineering, 51(4), $355-364$.

Defraeye, T. (2014). Advanced computational modelling for drying processes - A review. Applied Energy, 131, 323-344.

Dev, S. R. S., Geetha, P., Orsat, V., Gariépy, Y., \& Raghavan, G. S. V. (2011). Effects of microwave-assisted hot air drying and conventional hot air drying on the drying kinetics, color, rehydration, and volatiles of moringa oleifera. Drying Technology, 29, 1452-1458 (February 2014).

Dziak, J. (2008). Application of radio-frequency wave and micro-wave devices in drying and bleaching of wooden pulp. Applied Thermal Engineering, 28(10), 1189-1195.

El-mesery, H. S., \& Mwithiga, G. (2015). Performance of a convective, infrared and combined infrared- convective heated conveyor-belt dryer. Journal Food Science Technology, 52(5), 2721-2730.

El-sebaii, A. A., \& Shalaby, S. M. (2012). Solar drying of agricultural products: A review. Renewable and Sustainable Energy Reviews, 16(1), 37-43.

Erbay, Z., \& Icier, F. (2010). A review of thin layer drying of foods: Theory, modeling, and experimental results. Critical Reviews in Food Science and Nutrition, 50(5), $441-464$.

Fang, S., Wang, Z., Hu, X., Chen, F., Zhao, G., Liao, X., et al. (2011). Energy requirement and quality aspects of Chinese jujube (Zizyphus jujuba Miller) in hot air drying followed by microwave drying. Journal of Food Process Engineering, 34(2), $491-510$.

Fernandes, F, A. N., Linhares, F. E., \& Rodrigues, S. (2008). Ultrasound as pretreatment for drying of pineapple. Ultrasonics Sonochemistry, 15(6), 1049-1054.

Ganesapillai, M., Regupathi, I., \& Murugesan, T. (2011). Modeling of thin layer drying of banana (Nendran spp) under microwave, convective and combined microwave-convective processes. Chemical Product and Process Modeling, 6(1), $1-27$.

Gowen, A. A., Abu-Ghannam, N., Frias, J., \& Oliveira, J. (2008). Modeling dehydration and rehydration of cooked soybeans subjected to combined microwave-hot-air drying. Innovative Food Science and Emerging Technologies, 9(1), 129-137.

Hashim, N., Onwude, D., \& Rahaman, E. (2014). A preliminary Study: Kinetic model 
of drying process of pumpkins ( Cucurbita Moschata ) in a convective hot air dryer. Agriculture and Agricultural Science Procedia, 2(2), 345-352.

Hebbar, H. U., \& Rastogi, N. K. (2001). Mass transfer during infrared drying of cashew kernel. Journal of Food Engineering, 47(1), 1-5.

Hebbar, H. U., Vishwanathan, K. H., \& Ramesh, M. N. (2004). Development of combined infrared and hot air dryer for vegetables. Journal of Food Engineering, 65(4), 557-563.

Hemis, M., Choudhary, R., Gariepy, Y., \& Raghavan, V. G. S. (2015). Experiments and modelling of the microwave assisted convective drying of canola seeds. Biosystems Engineering, 139, 121-127.

Horrungsiwat, S., Therdthai, N., \& Ratphitagsanti, W. (2016). Effect of combined microwave-hot air drying and superheated steam drying on physical and chemical properties of rice. International Journal of Food Science \& Technology, $1-9$.

Jaturonglumlert, S., \& Kiatsiriroat, T. (2010). Heat and mass transfer in combined convective and far-infrared drying of fruit leather. Journal of Food Engineering, $100(2), 254-260$.

Jiao, A., Xu, X., \& Jin, Z. (2014). Modelling of dehydration-rehydration of instant rice in combined microwave-hot air drying. Food and Bioproducts Processing, 92(3), 259-265.

Karim, A., \& Hawlader, M. N. A. (2005). Drying characteristics of banana: Theoretical modelling and experimental validation. Journal of Food Engineering, 70, 35-45.

Kassem, A. S., Shokr, A. Z., El-Mahdy, A. R., Aboukarima, A. M., \& Hamed, E. Y. (2011). Comparison of drying characteristics of Thompson seedless grapes using combined microwave oven and hot air drying. Journal of the Saudi Society of Agricultural Sciences, 10(1), 33-40.

Kaur, K., \& Singh, A. K. (2014). Drying kinetics and quality characteristics of beetroot slices under hot air followed by microwave finish drying. African Journal of Agricultural Research, 9(12), 1036-1044.

Kaur, N., \& Singh, a. K. (2016). Ohmic Heating: Concept and applications- a review. Critical Reviews in Food Science and Nutrition, 56(14), 2338-2351.

Klemes, J., Smith, R., \& Kim, J. K. (2008). Preface. In J. Klemes, R. Smith, \& J. Kim (Eds.), Handbook of water and energy management in food processing. Boca Raton: CRC press.

Koumoutsakos, A., Avramidis, S., \& Hatzikiriakos, S. G. (2001). Radio frequency vacuum drying oF wood. II. Experimental model evaluation. Drying Technology, 19(1), 85-98.

Krishnamurthy, K., Khurana, H., Jun, S., Irudayaraj, J., \& Demirci, A. (2008). Infrared heating in food processing: An overview. Comprehensive Reviews in Food Science and Food Safety, 7(1), 2-13.

Łechtańska, J. M., Szadzińska, J., \& Kowalski, S. J. (2015). Microwave- and infraredassisted convective drying of green pepper: Quality and energy considerations. Chemical Engineering and Processing: Process Intensification, 98, 155-164.

Li, C., \& Lee, N.-H. (2008). The effect of compressive load on the moisture content of oak blocks during radio-frequency/vacuum drying. Forest Products Journal, 58(4), 34-38.

Liu, Y., Aziz, M., Fushimi, C., Kansha, Y., Mochidzuki, K., Kaneko, S., et al. (2012). Exergy analysis of biomass drying based on self-heat recuperation technology and its application to industry: A simulation and experimental study. Industrial and Engineering Chemistry Research, 51(30), 9997-10007.

Liu, Y., Aziz, M., Kansha, Y., Bhattacharya, S., \& Tsutsumi, A. (2014). Application of the self-heat recuperation technology for energy saving in biomass drying system. Fuel Processing Technology, 117, 66-74.

Luchese, C. L., Gurak, P. D., \& Marczak, L. D. F. (2015). Osmotic dehydration of physalis (Physalis peruviana L.): Evaluation of water loss and sucrose incorporation and the quantification of carotenoids. LWT - Food Science and Technology, 63(2), 1128-1136.

Madhava Naidu, M., Vedhashree, M., Satapathy, P., Khanum, H., Ramsamy, R., \& Umesh Hebbar, H. (2015). Effect of drying methods on the quality characteristics of Dill (Anethumgraveolens) greens. Food Chemistry, 192, 849-856.

Malafronte, L., Lamberti, G., Barba, A. A., Raaholt, B., Holtz, E., \& Ahrné, L. (2012). Combined convective and microwave assisted drying: Experiments and modeling. Journal of Food Engineering, 112(4), 304-312.

Marra, F., Zhang, L., \& Lyng, J. G. (2009). Radio frequency treatment of foods: Review of recent advances. Journal of Food Engineering, 91(4), 497-508.

Mihindukulasuriya, S. D. F., \& Jayasuriya, H. P. W. (2015). Drying of chilli in a combined infrared and hot air rotary dryer. Journal of Food Science and Technology, 52(8), 4895-4904.

Miura, N., Yagihara, S., \& Mashimo, S. (2003). Microwave dielectric properties of solid and liquid foods investigated by time-domain reflectometry. Journal of Food Science, 68(4), 1396-1403.

Mirzabeigi Kesbi, O., Sadeghi, M., \& Mireei, S. A. (2016). Quality assessment and modeling of microwave-convective drying of lemon slices. Engineering in Agriculture, Environment and Food, 9(3), 216-223.

Mongpraneet, S., Abe, T., \& Tsurusaki, T. (2004). Kinematic model for a far infrared vacuum dryer. Drying Technology, 22(7), 1675-1693.

Mongpraneet, S., Abe, T., Tsurusaki, T., Vol, A., Society, A., \& Engineers, A. (2016). Far infrared vacuum and convection drying of Welsh onion. Transaction of ASAE, 45(5), 1529.

Moses, J. A., Norton, T., Alagusundaram, K., \& Tiwari, B. K. (2014). Novel drying techniques for the food industry. Food Engineering Reviews, 6(3), 43-55.

Motevali, A., Minaei, S., Khoshtaghaza, M. H., \& Amirnejat, H. (2011). Comparison of energy consumption and specific energy requirements of different methods for drying mushroom slices. Energy, 36(11), 6433-6441.

Mujumdar, A. S., \& Law, C. L. (2010). Drying Technology: Trends and applications in postharvest processing. Food and Bioprocess Technology, 3(6), 843-852.

Murphy, A., Morrow, R., \& Besley, L. (1992). Combined radio-frequency and forcedair drying of alfalfa. Journal of Microwave Power and Electromagnetic Energy, 27(4), 223-232.

Nadi, F., Rahimi, G. H., Younsi, R., Tavakoli, T., \& Hamidi-Esfahani, Z. (2012). Numerical simulation of vacuum drying by Luikov's equations. Drying Technology, 30(2), 197-206.

Nathakaranakule, A., Jaiboon, P., \& Soponronnarit, S. (2010). Far-infrared radiation assisted drying of longan fruit. Journal of Food Engineering, 100(4), 662-668.

Nowak, D., \& Lewicki, P. P. (2004). Infrared drying of apple slices. Innovative Food Science and Emerging Technologies, 5(3), 353-360.

Nuthong, P., Achariyaviriya, A., Namsanguan, K., \& Achariyaviriya, S. (2011). Kinetics and modeling of whole longan with combined infrared and hot air. Journal of Food Engineering, 102(3), 233-239.

Onwude, D. I., Hashim, N., Janius, R. B., Nawi, N. M., \& Abdan, K. (2016a). Modeling the thin-layer drying of fruits and vegetables: A review. Comprehensive Reviews in Food Science and Food Safety, 15(3), 599-618.

Onwude, D. I., Hashim, N., Janius, R., Nawi, N., \& Abdan, K. (2016b). Evaluation of a suitable thin layer model for drying of pumpkin under forced air convection. International Food Research Journal, 23(3), 1173-1181.

Piyasena, P., Dussault, C., Koutchma, T., Ramaswamy, H. S., \& Awuah, G. B. (2003). Radio frequency heating of foods: Principles, applications and related properties-a review. Critical Reviews in Food Science and Nutrition, 43(6), 587-606.

Ponkham, K., Meeso, N., Soponronnarit, S., \& Siriamornpun, S. (2012). Modeling of combined far-infrared radiation and air drying of a ring shaped-pineapple with/ without shrinkage. Food and Bioproducts Processing, 90(2), 155-164.

Praveen Kumar, D. G., Hebbar, H. U., \& Ramesh, M. N. (2006). Suitability of thin layer models for infrared-hot air-drying of onion slices. LWT - Food Science and Technology, 39(6), 700-705.

Raghavan, G. S. V., Rennie, T. J., Sunjka, P. S., Orsat, V., Phaphuangwittayakul, W., \& Terdtoon, P. (2005). Overview of new techniques for drying biological materials with emphasis on energy aspects. Brazilian Journal of Chemical Engineering, 22(2), 195-201.

Raso, J., \& Barbosa-Cánovas, G. V. (2003). Nonthermal preservation of foods using combined processing techniques. Critical Reviews in Food Science and Nutrition, 43(3), 265-285.

Rastogi, N. K. (2012a). Infrared heating of fluid foods. In P. J. Cullen, B. K. Tiwari, \& V. P. Valdramidis (Eds.), Novel thermal and non-thermal technologies for fluid foods (pp. 411-432). San Diego: Academic Press [chapter 13].

Rastogi, N. K. (2012b). Recent trends and developments in infrared heating in food processing. Critical Reviews in Food Science and Nutrition, 52(9), 737-760.

Ratti, C., \& Mujumdar, A. (2014). Infrared drying. In Handbook of industrial drying, fourth edition (pp. 405-420). CRC Press.

Rawson, A., Patras, A., Tiwari, B. K., Noci, F., Koutchma, T., \& Brunton, N. (2011). Effect of thermal and non thermal processing technologies on the bioactive content of exotic fruits and their products: Review of recent advances. Food Research International, 44(7), 1875-1887.

Riadh, M. H., Ahmad, S. A. B., Marhaban, M. H., \& Soh, A. C. (2015). Infrared heating in food drying: An overview. Drying Technology, 33(3), 322-335.

Sadeghi, M., Mirzabeigi Kesbi, O., \& Mireei, S. A. (2013). Mass transfer characteristics during convective, microwave and combined microwave-convective drying of lemon slices. Journal of the Science of Food and Agriculture, 93(3), 471-478.

Sadin, R., Chegini, G., \& Khodadadi, M. (2014). Development and performance evaluation of a combined infrared and hot air dryer. Journal of Biological and Environmental Sciences, 8(22), 11-18.

Sagar, V. R., \& Suresh Kumar, P. (2010). Recent advances in drying and dehydration of fruits and vegetables: A review. Journal of Food Science and Technology, 47(1), $15-26$.

Sakai, N., \& Hanzawa, T. (1994). Applications and advances in far-infrared heating in Japan. Trends in Food Science and Technology, 5(11), 357-362.

Seremet (Ceclu), L., Botez, E., Nistor, O.-V., Andronoiu, D. G., \& Mocanu, G.-D. (2015). Effect of different drying methods on moisture ratio and rehydration of pumpkin slices. Food Chemistry, 195, 104-109.

Shi, J., Pan, Z., McHugh, T. H., Wood, D., Hirschberg, E., \& Olson, D. (2008). Drying and quality characteristics of fresh and sugar-infused blueberries dried with infrared radiation heating. LWT - Food Science and Technology, 41(10), 1962-1972.

Shinde, A., Das, S., \& Datta, A. K. (2013). Quality improvement of orthodox and CTC tea and performance enhancement by hybrid hot air-radio frequency (RF) dryer. Journal of Food Engineering, 116(2), 444-449.

Soysal, Y., Arslan, M., \& Keskin, M. (2009). Intermittent microwave-convective air drying of oregano. Food Science and Technology International, 15(4), 397-406.

Soysal, Y., Ayhan, Z., Eştürk, O., \& Arikan, M. F. (2009). Intermittent microwaveconvective drying of red pepper: Drying kinetics, physical (colour and texture) and sensory quality. Biosystems Engineering, 103(4), 455-463.

Talens, C., Castro-giraldez, M., \& Fito, P. J. (2016). LWT - Food science and technology study of the effect of microwave power coupled with hot air drying on orange peel by dielectric spectroscopy. LWT - Food Science and Technology, 66, 622-628.

Tuncel, N. B., Yilmaz, N., Kocabiyik, H., Öztürk, N., \& Tunçel, M. (2010). The effects of infrared and hot air drying on some properties of corn (Zea mays). Journal of Food, Agriculture and Environment, 8(1), 63-68.

Varith, J., Dijkanarukkul, P., Achariyaviriya, A., \& Achariyaviriya, S. (2007). Combined microwave-hot air drying of peeled longan. Journal of Food Engineering, 81(2), 459-468.

Venkatesh, M. S., \& Raghavan, G. S. V. (2004). An overview of microwave processing 
and dielectric properties of agri-food materials. Biosystems Engineering, 88(1), $1-18$.

Vicente, A., \& Castro, I. A. (2007). Novel thermal processing technologies. In G. Tewari, \& V. K. Juneja (Eds.), Advances in thermal and non-thermal food preservation (pp. 99-130). Ames, Iowa, USA: Blackwell Publishing.

Vishwanathan, K. H., Hebbar, H. U., \& Raghavarao, K. S. M. (2010). Hot air assisted infrared drying of vegetables and its quality. Food Science and Technology Research, 16(5), 381-388.

Wang, Y., Li, Y., Wang, S., Zhang, L., Gao, M., \& Tang, J. (2011). Review of dielectric drying of foods and agricultural products. International Journal of Biological and Agricultural Engineering, 4(1), 1-19.

Wang, H., Zhang, M., \& Mujumdar, A. S. (2014a). Comparison of three new drying methods for drying characteristics and quality of shiitake mushroom (Lentinus edodes ). Drying Technology, 32(15), 1791-1802.

Wang, Y., Zhang, L., Johnson, J., Gao, M., Tang, J., Powers, J. R., et al. (2014b) Developing hot air-assisted radio frequency drying for in-shell Macadamia nuts. Food and Bioprocess Technology, 7(1), 278-288.

Witrowa-Rajchert, D., Wiktor, A., Sledz, M., \& Nowacka, M. (2014). Selected emerging technologies to enhance the drying process: A review. Drying Technology: An International Journal, 32(11), 1386-1396.

Workneh, T. S., \& Oke, M. O. (2013). Thin layer modelling of microwave-convective drying of tomato slices. International Journal of Food Engineering, 9(1), 75-90.

Wray, D., \& Ramaswamy, H. S. (2015). Novel concepts in microwave drying of foods. Drying Technology, 33(7), 769-783.

Yousefi, A., Niakousari, M., \& Moradi, M. (2013). Microwave assisted hot air drying of papaya ( Carica papaya L.) pretreated in osmotic solution. African Journal of Agricultural Research, 8(25), 3229-3235.

Yuan, Y., Tan, L., Xu, Y., Yuan, Y., \& Dong, J. (2015). Optimization of combined drying for lettuce using response surface methodology. Journal of Food Processing and Preservation, $1-11$.

Zare, D., Naderi, H., \& Ranjbaran, M. (2015). Energy and quality attributes of combined hot-air/infrared drying of paddy. Drying Technology, 33(5), 570-582.

Zhang, M., Tang, J., Mujumdar, A. S., \& Wang, S. (2006). Trends in microwave-related drying of fruits and vegetables. Trends in Food Science \& Technology, 17(10), $524-534$.

Zhang, M., Chen, H., Mujumdar, A. S., Tang, J., Miao, S., \& Wang, Y. (2015). Recent developments in high-quality drying of vegetables, fruits and aquatic products. Critical Reviews in Food Science and Nutrition.

\section{Glossary}

$M_{0}$ : Initial moisture content ( $\mathrm{g}$ water/ $\mathrm{g}$ dry solid)

$M_{e}$ : Equilibrium moisture content ( $\mathrm{g}$ water/g dry solid)

$M C$ : Moisture content (g water/g dry solid)

$S P$ : Optimum selection percentage (\%)

$R R$ : Rehydration ratio

$A H$ : Absolute humidity $\left(\mathrm{g} / \mathrm{m}^{3}\right)$

$t$ : Time (s)

W: Width (m or $\mathrm{mm}$ )

DM: Dry matter

$R C$ : Rehydration capacity
$P$ : Power $(\mathrm{kW})$

SEC: Specific energy consumption

$P R$ : Pulse ratio

$D R$ : Drying rate $(\mathrm{kg} / \mathrm{kg} \mathrm{min})$

$A D R$ : Average drying rate $(\mathrm{kg} / \mathrm{kg} \mathrm{min})$

$V_{s}$ : Sample volume $\left(\mathrm{m}^{3}\right)$

$V_{a}$ : Ambient air velocity $(\mathrm{m} / \mathrm{s})$

$h$ : Thickness (mm)

$L$ : Length $(\mathrm{mm})$

A: Wavelength $(\mu \mathrm{m})$

$f$ : Frequency $(\mathrm{Hz})$

$d_{e}$ : Distance between IR emitter and sample $(\mathrm{cm})$

$T$ : Temperature $\left({ }^{\circ} \mathrm{C}\right)$

$T_{a}$ : Ambient temperature $\left({ }^{\circ} \mathrm{C}\right)$

$v$ : Air velocity $(\mathrm{m} / \mathrm{s})$

$R H$ : Relative humidity (\%)

$H A D$ : Hot-air-drying or Hot-air-dryer

HA: Hot-air

$M W D$ : Microwave drying

$M W$ : Microwave

IR: Infrared

IRD: Infrared drying

$V D$ : Vacuum drying

HP: Heat pump

IRP: Infrared power $(\mathrm{W})$

IRI: Infrared intensity $\left(\mathrm{W} / \mathrm{m}^{2}\right)$

$P_{d}$ : Power density $(\mathrm{W})$

$P$ : Power intensity $\left(\mathrm{W} / \mathrm{m}^{2}\right)$

SR: Shrinkage ratio

$L^{*}$ : Lightness index

$a^{*}$ : Redness index

$b^{*}$ : Yellowness index

$\triangle E$ : Total colour change

$D_{\text {eff: }}$ Effective moisture diffusivity $\left(\mathrm{m}^{2} / \mathrm{s}\right)$

$E_{a}$ : Activation energy $(\mathrm{kJ} / \mathrm{mol})$

BI: Brownness index

SMIR: Short-medium- infrared radiation

VC: Vitamin C

$H R Y$ : Head rice yield

SFR: Shear force ratio

MIR: Mid-infrared radiation

FIR: Far-infrared radiation

$R F$ : Radio frequency

$R F D$ : Radio-frequency drying

$E$ : Electric field strength $(\mathrm{V} / \mathrm{cm})$

$h_{c}$ : Cylindrical height ( $\mathrm{m}$ or $\mathrm{mm}$ )

Pt: Pulse time (s)

Rt: Recovery time (s)

$I H$ : Inductive heating

$N$ : Pulse number

EM: Electromagnetic

CIR: Catalytic infrared 